EISSN: 2706-7955 ISSN: 2077-4605

DOI: 10.36632/mejar/2021.10.2.40

Journal homepage: www.curresweb.com

Pages: 548-562

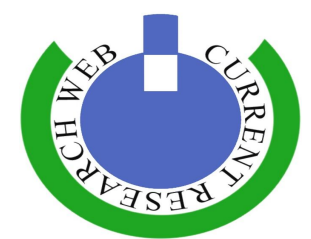

\title{
Effect of Some Microelements and Bio-Stimulants and Their Interaction on Calendula Officinalis Plants in Sandy Soils
}

\author{
Taghreed E. Eissa ${ }^{1}$ and Alaa eldeen A. Shaheen ${ }^{2}$
}

${ }^{1}$ Ornamental Plants and Landscape Gardening Res. Dept., Hort. Res. Inst., Agric. Res. Center, Giza, Egypt.

${ }_{2}^{2}$ Sandy and Limestone Lands Dept., Water and Environment Res. Inst., Agric. Res. Center, Giza, Egypt. Received: 23 March $2021 \quad$ Accepted: 25 April $2021 \quad$ Published: 05 May 2021

\begin{abstract}
The present work was carried out at the Experimental farm in Ismailia Research Station, Agric. Res. Center, Egypt, during 2019 and 2020 seasons. The investigation aimed to improve plant quality and productivity of Calendula officinalis plants in sandy soil. Iron application at the rate of $(0,50$ and 100 $\mathrm{ppm})$ as foliar aqueous solution $\left(\mathrm{Fe} \mathrm{SO}_{4} \cdot 3 \mathrm{H}_{2} \mathrm{O}\right)$ and copper application at the rate of $(0,25$ and $50 \mathrm{ppm})$ as foliar aqueous solution $\left(\mathrm{Cu} \mathrm{SO}_{4} \cdot \mathrm{H}_{2} \mathrm{O}\right)$ while, yeast and amino acids were applied at the rate of $2 \mathrm{~g} / \mathrm{l}$. The treatments were applied three times, the first time at 30 days after sowing; the second is twenty days after the first application at stem elongation and the third before flowering stages. The results indicated that, the application of either microelement i.e. ( $\mathrm{Fe}$ and $\mathrm{Cu}$ ) treatments and bio-stimulants i.e. (yeast and amino acids) or coupled gave significant increased on vegetative growth and flowers parameters under study as compared with untreated plants in both seasons. The application of the treatment of $100 \mathrm{ppm}$ of iron and $50 \mathrm{ppm}$ copper coupled with amino acid at $2 \mathrm{~g} / 1$ improved growth performance, flowering traits and chemical constituents (total chlorophyll in leaves, beta carotenoids, total flavonoids in dry ray flowers, nitrogen, phosphorus and potassium percentage, iron, copper and zinc (ppm) in leaves) as compared with other treatments.
\end{abstract}

Keywords: Iron (Fe), Copper (Cu), Bio-stimulators, foliar spray, and Calendula officinalis plants

\section{Introduction}

Calendula officinalis L. commonly known as pot marigold, English marigold, butterwort, Bride of the Sun and bull flower, it is an annual medicinal and ornamental plant, it is a beautiful marketable flower of Asteraceae family. Marigold is used as cut flower and in garden displays, garlands, bouquets and stage decorations. Marigold is internationally known for its medicinal position containing various phyto-chemicals including fatty acids, carbohydrates, amino acids lipids, terpenoids, flavonoids, coumarins and quinones and the oil of Calendula contains limonene, linalool and linalylacetate. It's phyto-chemicals showing some important biological activities like wound healing, immuno-stimulant, spasmogenic and spasmolytic, hepatoprotective, anti-amylase, anti-fungal, antidiabetic, anti-bacterial and anti-cancerous. It is used for more for medicinal than culinary purposes, the yellow petals are an excellent remedy for red and inflamed skin, also, their antiseptic and medicinal properties serving in stopping the spread of infection, and speed up the rate of healing. Calendula cream is good for acne and diaper rash treatment of skin disorders and pain, and as a bactericide, antiseptic .These plants have a long history of usage as anti-inflammatory, antigenotoxic, chemoprotective, antitumor, antibacterial, antiseptic properties and show a major role in human health by combating injury caused by oxidizing agents.. The Flavonoids compounds, one of pigments classes in C. officinalis, have antioxidant activities and the petals and pollen grains of Calendula contain, triterpenoid esters, carotenoids, flavaxanthin, auroxanthin, and compounds that are used in industrial paints and industrial nylon (Yoshikawa et al., 2001, Hamburger et al., 2003, Naguib et al., 2005, Bashir et al., 2006, Ukiya et al., 2006, Bolderston et al., 2006, Fonseca et al., 2010, Bunghez and Ion, 2011, Borghei et al., 2011, Khan et al., 2011, Rafiee

Corresponding Author: Taghreed E. Eissa, Ornamental Plants and Landscape Gardening Res. Dept., Hort. Res. Inst., Agric. Res. Center, Egypt. E-mail: dr.taghreed.eissa.2019@gmail.com 
et al., 2013, Abou-Sreea and Yassen, 2016, Jan et al., 2017, Ashwlayan et al., 2018 and Hussain et al., 2020).

In sandy soils, nutrient concentrations may not be sufficient and therefore, micro-nutrients are loss in these soils quickly so that the roots of the plants cannot absorb them from the soil and some nutrients do not transit to leaves. So, foliar fertilization is a particularly useful method of micro-nutrients could resolve this problem and decrease soil fertilizer loss, foliar nurturing is a way to decline the use of chemical fertilizers and reduce environmental dangers, especially now to advance fertilizer use in the world is a main strategy. It can be designed to meet plants specific wants for one or more micro- or macro-nutrients, especially trace minerals, and to correct deficiencies, strengthen weak or damaged yields, accelerate growth, and grow healthier and better plants. Applications of micronutrients are more suitable than the soil application, because of the rapid overcoming on deficient, easy to use, decrease the toxicity caused by addition and avoid of elements stabilization in the soil and avoiding losses through fixation. It's well-known that most cultivated zones in Egypt appeared symptoms of deficiency of $\mathrm{Mn}, \mathrm{Zn}, \mathrm{Fe}, \mathrm{Cu}$ and $\mathrm{Mg}$. Therefore, the beneficial effects of micronutrients are acute for normal growth of plants, and involved in all metabolic and cellular function. Iron and Copper are well known vital micronutrients, which play an important role in vegetative and reproductive cycle of plants. $\mathrm{Fe}$ is also essential micro nutrients which plays a vital role in the respiration and photosynthesis and also affects its properties. Also, Copper is necessary microelement in higher plants as it occurs as part of the prosthetic groups of several enzymes. It was shown to be associated with proteins or nuclear contaminants (Sunitha, 2006, Broadley et al., 2007, Heidari et al., 2008, Hassegawa et al., 2008, Singh et al., 2012, Younis et al., 2013, Yadegari, 2013b and Shahram et al., 2015).

Bio-stimulators as natural substances encourage metabolism and metabolic processes, which can be increase plants yield and secondary metabolites content. The maximum currently used bio-stimulators are amino acid substances. Numerous amino acids moreover act as precursors of other nitrogen containing compounds, e.g., nucleic acids. Amino acids can play wide roles in plants including acting as regulatory and signaling molecules. In current years, there is a growing attention with natural bio stimulants including yeast extract, it is rich with a mixture of amino acids, peptides, the best sources of the B-complex vitamins such as B1, B2, B6 and B12, carbohydrates and valuable source of phytohormones especially, cytokinins that increase cell division and enlargement (Rai, 2002, Rafiee et al., 2013 and Nofal, et al., 2015).

For the sake of studying the effect of foliar spray of $\mathrm{Fe}, \mathrm{Cu}$, yeast and amino acid as individually or combined treatments on the growth, flowering and some chemical constituents of Calendula plants the current work was carried out.

\section{Materials and Methods}

To achieve the objective of this study, a field experiment was conducted during two successive seasons (2019 and 2020) at the Experimental farm in Ismailia Research Station Egypt to study the effect of foliar spray of $\mathrm{Fe}, \mathrm{Cu}$, yeast and amino acids as individually or mixed with other treatments on the growth, flowering and some chemical elements of Calendula officinalis plants, aiming to obtain high quality of flowers and productivity. Soil samples were taken from the soil surface $(0-30 \mathrm{~cm})$ and the main physical and chemical soil properties of the experimental field were determined according to the methods described by Klute (1986) and Page et al. (1982), Fe, $\mathrm{Mn}$, and $\mathrm{Cu}$ were extracted by DTPA according to Soltanpour and Schwab (1977) and were determined by using Atomic Absorption Spectorphotometer and are given in Table 1.The irrigation water analysis was showed in Table 2. The details of amino acids formulations are mentioned in Table 3.

\subsection{Plant material}

The seeds of Calendula plants were kindly obtained by the Orman garden Giza, Egypt and sown in nursery on $17^{\text {th }}$ Sept. 2019 and 2020 seasons. 


\subsection{Experimental design and treatments}

Twenty nine treatments were arranged as a factorial design on the base of completely randomized blocks with three replications. The plot area was $(10 \times 2)=20 \mathrm{~m}^{2}$ and included three ridges, each ridge was $50 \mathrm{~cm}$ apart and $10 \mathrm{~m}$ in length. Sowing was conducted manual, plant spacing in the each row was $5 \mathrm{~cm}$, and two seeds were put in the soil. In the 3-5 leaves phase plants were thin out to final row distance $(10 \mathrm{~cm})$. During the vegetation, used practical measures were standard (3 earth up and pest protection). All Agricultural practices necessary for seedlings production were achieved.

Table 1: Some characteristics of the experimental site

\begin{tabular}{|c|c|c|c|}
\hline Soil characteristics & Value & Soil characteristics & Value \\
\hline Particle size distribution \% & & Soluble cations in saturation extract 1: $5\left(\mathrm{mmole}^{-1}\right)$ & \\
\hline Coarse sand & 62.75 & $\mathrm{Ca}^{2+}$ & 2.86 \\
\hline Fine sand & 25.66 & $\mathrm{Mg}^{2+}$ & 2.15 \\
\hline Silt & 7.11 & $\mathrm{Na}^{+}$ & 1.34 \\
\hline Clay & 4.48 & $\mathrm{~K}^{+}$ & 2.05 \\
\hline \multicolumn{4}{|l|}{ Textural class: sandy } \\
\hline $\mathbf{p H}$ & 7.38 & Soluble anions in saturation extract 1: $5\left(\mathrm{mmole}^{-1}\right)$ & \\
\hline $\mathrm{EC}\left(\mathrm{dSm}^{-1}\right)$ & 0.84 & $\mathrm{CO}_{3}^{2-}$ & 0.00 \\
\hline $\mathrm{CaCO}_{3} \%$ & 0.15 & $\mathrm{HCO}_{3}^{-}$ & 4.18 \\
\hline Organic matter \% & 0.25 & $\mathrm{Cl}^{-}$ & 1.22 \\
\hline & & $\mathrm{SO}_{4}^{-2}$ & 3.00 \\
\hline Available nutrients $\left(\mathrm{mg} \mathrm{Kg}^{-1}\right)$ & & Available nutrients $\left(\mathrm{mg} \mathrm{Kg}^{-1}\right)$ & \\
\hline $\mathbf{N}$ & 44.00 & $\mathrm{Fe}$ & 0.45 \\
\hline $\mathbf{P}$ & 2.43 & $\mathrm{Mn}$ & 0.31 \\
\hline $\mathbf{K}$ & 168 & $\mathrm{Zn}$ & 0.25 \\
\hline
\end{tabular}

Table 2: The irrigation water analysis in Ismailia Research Station.

\begin{tabular}{|c|c|}
\hline Characters & Value \\
\hline pH & 7.84 \\
\hline$E C\left(d S ~ m^{-1}\right)$ & 0.72 \\
\hline \multicolumn{2}{|c|}{ Soluble cations (meq/L) } \\
\hline $\mathrm{Ca}^{2+}$ & 2.99 \\
\hline $\mathbf{M g}^{2+}$ & 1.55 \\
\hline $\mathrm{Na}$ & 1.80 \\
\hline $\mathbf{K}$ & 0.50 \\
\hline \multicolumn{2}{|c|}{ Soluble anions (meq/L) } \\
\hline $\mathrm{CO}_{3}^{2-}$ & 0.00 \\
\hline $\mathrm{HCO}_{3}^{-}$ & 3.20 \\
\hline $\mathrm{Cl}^{-}$ & 1.20 \\
\hline $\mathrm{SO}_{4}{ }^{2-}$ & 2.44 \\
\hline
\end{tabular}

Table 3: Formulation of amino acids used in the experimental treatments.

\begin{tabular}{lc}
\hline Amino acids & Concentrations \% \\
\hline Arginine & 5.50 \\
Aspatic & 7.50 \\
Alanine & 13.00 \\
Sarine & 5.00 \\
Proline & 8.00 \\
Glycline & 19.00 \\
Glutamic & 8.00 \\
Lysine & 5.20 \\
\hline
\end{tabular}

\subsection{Treatments}

Aqueous solution of $\mathrm{FeSO}_{4} \cdot 3 \mathrm{H}_{2} \mathrm{O}(\mathrm{Fe} 20 \%)$ was applied at the rate of $(0,50$ and $100 \mathrm{ppm})$ and $\mathrm{CuSO}_{4} \cdot \mathrm{H}_{2} \mathrm{O}(\mathrm{Cu} 23 \%)$ was applied at the rate of $(0,25$ and $50 \mathrm{ppm})$ and yeast and amino acids were applied at $2 \mathrm{~g} / \mathrm{l}$. Yeast (Saccharomyces cerevisiae): active dry yeast was obtained and $2 \mathrm{~g}$ of yeast was 
weighed and put with $100 \mathrm{~cm}$ of water in a glass 3 beaker. A teaspoon full of sugar was added. The beaker was kept in a dark warm place for 30 minutes. Contents of the beaker were then filtered into a one liter measuring flask and water was added to one liter final volume before application on the plants. The treatments were applied three times on the plants; the first time at 30 days after sowing, the second is twenty days after the first application at stem elongation and the third before flowering stages. Few drops of Tween 20 were added to the spray solution to serve as a wetting agent. Sprays were applied using a hand pressure sprayer. The volume of the spraying solution was maintained just to cover completely the plant foliage until drip. All the plants received normal agriculture practices whenever they needed. In addition, they received uniform treatments of manure $15 \mathrm{~m}^{3}$ per feddan., irrigation and fertilization at the field according to common practices i.e. Calendula plants fertilized with $300 \mathrm{~kg}$ per feddan ammonium sulphate $(20.5 \% \mathrm{~N}), 300 \mathrm{~kg}$ per feddan calcium super phosphate $\left(15.5 \% \mathrm{P}_{2} \mathrm{O}_{2}\right)$ and $100 \mathrm{~kg}$ per feddan potassium sulphate $\left(48 \% \mathrm{~K}_{2} \mathrm{O}\right)$. Half of the $\mathrm{N}$ and $\mathrm{K}$ rates were added after 30 days from sowing and the second application was done after 30 days from the first application. Application of calcium super phosphate was done as one dose during the preparation of the soil. All agriculture practices operations other than experimental treatments necessary for growth and development as cultivation, irrigation and pest control were followed whenever it was necessary and were done according to the recommendations of Ministry of Agriculture, Egypt (Abou-Sreea and Yassen, 2016).

\subsection{Data recorded}

Studied Characters: after 85 days from sowing at full blooming, ten plants were chosen from each treatment to determine the studied characters.

\subsection{Morphological Characters:}

Plant height $(\mathrm{cm})$, number of branches per plant, fresh and dry weight of plant $(\mathrm{g})$ number of leaves per plant, root length, fresh and dry weight of roots $(\mathrm{g})$, flowers number per plant,Flower head diameter $(\mathrm{cm})$ and vase life.

\subsection{Chemical constituents:} (1982).

1- Total chlorophyll in leaves were determined using the method described by according to Moran

2. Pigments in dry flowers (Beta-carotene) $\mathrm{mg} / \mathrm{g}$ : the pigments were determined at last collection according to method described by AOAC (1970).

4. Total flavonoids (mg/g d.w.): were determined in dried flower-head according to Bacot (1954).

4. Total N, $\mathrm{P}$ and $\mathrm{K} \%$ in leaves were determined according to the methods of the (Cottenie et al., 1982). Iron, copper and Manganese (ppm) were determined using atomic absorption spectrophotometer to method described by (Cottenie et al., 1982).

\subsection{Statistical analysis:}

Data were tabulated and subjected to analysis of variance as a factorial experiment using MSTATC statistical software (1985) and Means of treatments were compared by Duncan's Multiple Range Test at 5\% level as indicated by Snedecor and Cochran (1980).

\section{Results and Discussion}

\subsection{Effect of foliar application of micro nutrients, bio-stimulators and their interaction on vegetative growth and flowers parameters in Calendula plants:}

Results showed that micronutrient is beneficial to pot marigold plants (Table, 4, 5, 6, 7 and 8), it is obvious that foliar application of Iron and Copper with yeast and amino acid gave significant increased on all vegetative growth and flowering parameters under study as compared with control plants in both seasons. From the present data we conclude that applications of Iron at $100 \mathrm{ppm}$ and 
Table 4: Effect of micro nutrients, bio-stimulators and their interaction on plant height and number of branches / plant of Marigold (Calendula officinalis L.) during (2019 and 2020) seasons.

\begin{tabular}{|c|c|c|c|c|c|c|c|c|c|}
\hline \multicolumn{2}{|l|}{ Treatments } & \multicolumn{4}{|c|}{ Plant height ( cm) } & \multicolumn{4}{|c|}{ Number of branches/ plant } \\
\hline \multirow{3}{*}{ Micro nutrients } & \multirow{3}{*}{$\begin{array}{l}\text { Concentrations } \\
\text { (ppm) }\end{array}$} & \multicolumn{4}{|c|}{$1^{\text {st }}$ season } & \multicolumn{4}{|c|}{$1^{\text {st }}$ season } \\
\hline & & \multicolumn{3}{|c|}{ Bio -stimulators $(\mathrm{g} / \mathrm{l})$} & \multirow[t]{2}{*}{ Mean B } & \multicolumn{3}{|c|}{ Bio -stimulators $(\mathrm{g} / \mathrm{l})$} & \multirow[t]{2}{*}{ Mean B } \\
\hline & & Control & Yeast & Amino acid & & Control & Yeast & Amino acid & \\
\hline Control & & $43.22 \mathrm{w}$ & $49.00 \mathrm{t}$ & 53.00 o & $48.41 \mathbf{H}$ & $10.23 \mathrm{z}$ & $11.23 \mathrm{y}$ & $13.00 \mathrm{u}$ & $11.49 \mathbf{I}$ \\
\hline $\mathbf{F e}$ & 50 & $50.65 \mathrm{r}$ & $52.02 \mathrm{q}$ & $55.00 \mathrm{~m}$ & $52.56 \mathbf{F}$ & $12.41 \mathrm{~V}$ & $13.54 \mathrm{r}$ & 15.231 & $13.73 \mathbf{F}$ \\
\hline $\mathbf{F e}$ & 100 & $53.00 \mathrm{o}$ & $56.20 \mathrm{k}$ & $59.00 \mathrm{i}$ & $56.07 \mathbf{E}$ & $13.23 \mathrm{t}$ & $14.41 \mathrm{p}$ & $16.24 \mathrm{j}$ & $14.63 \mathbf{E}$ \\
\hline $\mathbf{C u}$ & 25 & $42.32 \times$ & $45.00 \mathrm{v}$ & $50.00 \mathrm{~s}$ & $45.77 \mathbf{I}$ & $10.23 \mathrm{z}$ & $12.23 \mathrm{~W}$ & $14.64 \mathrm{o}$ & $12.37 \mathbf{H}$ \\
\hline $\mathrm{Cu}$ & 50 & $45.00 \mathrm{~V}$ & $48.00 \mathrm{u}$ & 56.001 & $49.67 \mathbf{G}$ & $11.42 \mathrm{x}$ & $14.25 \mathrm{q}$ & $15.12 \mathrm{~m}$ & $13.60 \mathbf{G}$ \\
\hline $\mathrm{Fe}+\mathrm{Cu}$ & $50+25$ & $53.20 \mathrm{n}$ & $57.15 \mathrm{j}$ & $60.42 \mathrm{~h}$ & $56.92 \mathbf{D}$ & $13.52 \mathrm{~s}$ & $16.20 \mathrm{k}$ & $19.50 \mathrm{e}$ & $16.41 \mathbf{D}$ \\
\hline $\mathbf{F e}+\mathbf{C u}$ & $\mathbf{5 0}+\mathbf{5 0}$ & $52.23 \mathrm{p}$ & $63.14 \mathrm{~g}$ & $72.15 \mathrm{e}$ & $62.51 \mathrm{C}$ & $14.65 \mathrm{n}$ & $17.53 \mathrm{~h}$ & $21.52 \mathrm{c}$ & $17.90 \mathrm{C}$ \\
\hline $\mathbf{F e}+\mathbf{C u}$ & $100+25$ & 56.001 & $75.00 \mathrm{~d}$ & $78.26 \mathrm{~b}$ & $69.75 \mathbf{B}$ & $16.45 \mathrm{i}$ & $19.16 \mathrm{f}$ & $23.46 \mathrm{~b}$ & $18.70 \mathbf{B}$ \\
\hline $\mathrm{Fe}+\mathrm{Cu}$ & $100+50$ & $63.23 \mathrm{f}$ & $78.00 \mathrm{c}$ & $80.60 \mathrm{a}$ & $73.94 \mathbf{A}$ & $18.23 \mathrm{~g}$ & $20.00 \mathrm{~d}$ & $26.25 \mathrm{a}$ & $21.49 \mathbf{A}$ \\
\hline \multirow[t]{2}{*}{ Mean A } & & $50.98 \mathrm{C}$ & $58.17 \mathbf{B}$ & $62.71 \mathrm{~A}$ & & $13.37 \mathrm{C}$ & $15.39 \mathbf{B}$ & $18.33 \mathbf{A}$ & \\
\hline & & \multicolumn{4}{|c|}{$2^{\text {nd }}$ season } & \multicolumn{4}{|c|}{$2^{\text {nd }}$ season } \\
\hline Control & & $46.11 \mathrm{z}$ & $54.65 \mathrm{t}$ & $59.12 \mathrm{n}$ & $53.29 \mathbf{H}$ & $9.00 \mathrm{z}$ & $12.45 \mathrm{u}$ & $14.12 \mathrm{r}$ & $11.86 \mathbf{I}$ \\
\hline $\mathbf{F e}$ & 50 & $57.02 \mathrm{~s}$ & 58.64 o & $61.23 \mathrm{~m}$ & $58.96 \mathbf{F}$ & $11.02 \mathrm{x}$ & $14.42 \mathrm{q}$ & $15.98 \quad 1$ & $13.81 \mathbf{G}$ \\
\hline Fe & 100 & $58.32 \mathrm{p}$ & $63.15 \mathrm{k}$ & $63.51 \mathrm{j}$ & $61.66 \mathbf{E}$ & $12.11 \mathrm{w}$ & $15.78 \mathrm{~m}$ & $17.14 \mathrm{i}$ & $15.01 \mathbf{E}$ \\
\hline $\mathrm{Cu}$ & 25 & $46.15 \mathrm{y}$ & $52.2 \mathrm{w}$ & $54.23 \mathrm{~V}$ & $50.86 \mathbf{I}$ & $10.42 \mathrm{y}$ & $13.75 \mathrm{t}$ & $15.00 \quad$ o & $13.06 \mathrm{H}$ \\
\hline $\mathrm{Cu}$ & 50 & $50.34 \mathrm{x}$ & $54.61 \mathrm{u}$ & $58.12 \mathrm{q}$ & $54.36 \mathbf{G}$ & $12.31 \mathrm{v}$ & $15.64 \mathrm{n}$ & 15.981 & $14.64 \mathrm{~F}$ \\
\hline $\mathbf{F e}+\mathbf{C u}$ & $50+25$ & $57.14 \mathrm{r}$ & $67.5 \mathrm{~h}$ & $69.64 \mathrm{f}$ & $64.76 \mathrm{D}$ & $13.97 \mathrm{~s}$ & $17.00 \mathrm{k}$ & $20.54 \mathrm{e}$ & $17.17 \mathbf{D}$ \\
\hline $\mathrm{Fe}+\mathrm{Cu}$ & $\mathbf{5 0}+\mathbf{5 0}$ & 58.64 o & $69.45 \mathrm{~g}$ & $72.64 \mathrm{~d}$ & $66.91 \mathrm{C}$ & $14.78 \mathrm{p}$ & $18.31 \mathrm{~h}$ & $21.42 \mathrm{~d}$ & $18.17 \mathrm{C}$ \\
\hline $\mathrm{Fe}+\mathrm{Cu}$ & $100+25$ & 61.501 & $72.11 \mathrm{e}$ & $74.56 \mathrm{c}$ & 69.39 В & $17.03 \mathrm{j}$ & $19.41 \mathrm{~g}$ & $23.89 \mathrm{~b}$ & 20.11 B \\
\hline $\mathrm{Fe}+\mathrm{Cu}$ & $100+50$ & $65.85 \mathrm{i}$ & $75.98 \quad b$ & 78.74 a & $73.52 \mathbf{A}$ & $19.45 \mathrm{f}$ & $21.45 \mathrm{c}$ & $25.16 \mathrm{a}$ & $22.02 \mathrm{~A}$ \\
\hline Mean A & & $55.67 \mathrm{C}$ & 63.14 B & $65.75 \mathrm{~A}$ & & $13.34 \mathrm{C}$ & 16.47 B & $18.80 \mathrm{~A}$ & \\
\hline
\end{tabular}

Means followed by the same letters in a column or raw do not differ significantly according to Duncan's New Multiple Range test at $\mathrm{P}=0.05$. 
Middle East J. Agric. Res., 10(2): 548-562, 2021

Table 5: Effect of micro nutrients, bio -stimulators and their interaction on fresh and dry weight of plant of Marigold (Calendula officinalis L.) during (2019 and 2020) seasons.

\begin{tabular}{|c|c|c|c|c|c|c|c|c|c|c|}
\hline \multirow{2}{*}{\multicolumn{2}{|c|}{ Treatments }} & \multicolumn{5}{|c|}{ Fresh weight of plant (g/plant) } & \multicolumn{4}{|c|}{ Dry weight of plant (g/plant) } \\
\hline & & \multicolumn{5}{|c|}{$1^{\text {st }}$ season } & \multicolumn{4}{|c|}{$1^{\text {st }}$ season } \\
\hline \multirow[t]{2}{*}{ Micro nutrients } & \multirow{2}{*}{$\begin{array}{l}\text { Concentrations } \\
\text { (ppm) }\end{array}$} & \multicolumn{4}{|c|}{ Bio-stimulators (g/l) } & \multirow[t]{2}{*}{ Mean B } & \multicolumn{3}{|c|}{ Bio -stimulators (g/l) } & \multirow[t]{2}{*}{ Mean B } \\
\hline & & Control & Yeast & & Amino acid & & Control & Yeast & Amino acid & \\
\hline Control & & $196.52 \mathrm{z}$ & 202.14 & $\mathrm{y}$ & $234.00 \mathrm{u}$ & 210.89 I & $14.80 \mathrm{z}$ & $15.22 \mathrm{y}$ & $17.62 \mathrm{u}$ & $15.88 \mathbf{I}$ \\
\hline $\mathbf{F e}$ & 50 & $223.38 \mathrm{~V}$ & 243.72 & $\mathrm{r}$ & $274.14 \quad 1$ & $247.08 \mathbf{F}$ & $16.82 \mathrm{v}$ & $18.35 \mathrm{~s}$ & 20.641 & $18.60 \mathbf{F}$ \\
\hline $\mathbf{F e}$ & 100 & $238.14 \mathrm{t}$ & 259.38 & $\mathrm{p}$ & $292.32 \mathrm{j}$ & $263.28 \mathbf{E}$ & $17.93 \mathrm{t}$ & $19.53 \mathrm{p}$ & $22.09 \mathrm{j}$ & $19.85 \mathbf{E}$ \\
\hline $\mathrm{Cu}$ & 25 & $196.54 \mathrm{z}$ & 220.14 & $\mathrm{w}$ & $263.52 \mathrm{o}$ & $226.73 \mathbf{H}$ & $14.82 \mathrm{z}$ & $16.58 \mathrm{w}$ & 19.84 o & $17.10 \mathbf{H}$ \\
\hline $\mathrm{Cu}$ & 50 & $205.56 \mathrm{x}$ & 256.50 & $\mathrm{q}$ & $272.16 \mathrm{~m}$ & $244.74 \mathbf{G}$ & $15.58 \mathrm{x}$ & $19.38 \mathrm{q}$ & $20.5 \mathrm{~m}$ & $18.49 \mathbf{G}$ \\
\hline $\mathbf{F e}+\mathbf{C u}$ & $50+25$ & $243.36 \mathrm{~s}$ & 291.60 & $\mathrm{k}$ & $351.00 \mathrm{e}$ & 295.32 D & $18.37 \mathrm{r}$ & $21.96 \mathrm{k}$ & $26.43 \mathrm{e}$ & $22.25 \mathbf{D}$ \\
\hline $\mathbf{F e}+\mathbf{C u}$ & $\mathbf{5 0}+\mathbf{5 0}$ & $263.70 \mathrm{n}$ & 315.54 & $\mathrm{~g}$ & $387.36 \mathrm{c}$ & $322.20 \mathrm{C}$ & $19.86 \mathrm{n}$ & $23.76 \mathrm{~g}$ & $29.17 \mathrm{c}$ & $24.26 \mathrm{C}$ \\
\hline $\mathbf{F e}+\mathbf{C u}$ & $100+25$ & $296.10 \mathrm{~h}$ & 295.64 & $\mathrm{i}$ & $422.28 \mathrm{~b}$ & $338.01 \quad \mathbf{B}$ & $22.34 \mathrm{~h}$ & $22.26 \mathrm{i}$ & $32.80 \mathrm{~b}$ & $25.80 \mathrm{~B}$ \\
\hline $\mathbf{F e}+\mathbf{C u}$ & $100+50$ & $328.14 \mathrm{f}$ & 360.00 & & $472.5 \mathrm{a}$ & $386.88 \mathbf{A}$ & $24.71 \mathrm{f}$ & $27.11 \mathrm{~d}$ & $35.58 \mathrm{a}$ & $29.13 \mathbf{A}$ \\
\hline \multirow[t]{2}{*}{ Mean A } & & $243.49 \mathrm{C}$ & 271.631 & & $329.92 \mathbf{A}$ & & $18.36 \mathbf{C}$ & 20.46 B & $24.96 \mathbf{A}$ & \\
\hline & & \multicolumn{5}{|c|}{$2^{\text {nd }}$ season } & \multicolumn{4}{|c|}{$2^{\text {nd }}$ season } \\
\hline Control & & $200.52 \mathrm{z}$ & 208.14 & $\mathrm{x}$ & $242.13 \mathrm{q}$ & 216.93 I & $15.08 \mathrm{z}$ & $15.67 \mathrm{x}$ & $18.53 \mathrm{u}$ & 16.43 I \\
\hline $\mathrm{Fe}$ & 50 & $227.38 \mathrm{u}$ & 249.72 & $\mathrm{r}$ & $282.14 \mathrm{k}$ & $253.08 \mathbf{F}$ & $18.12 \mathrm{v}$ & $19.87 \mathrm{q}$ & $21.35 \mathrm{~m}$ & $19.78 \mathrm{~F}$ \\
\hline $\mathrm{Fe}$ & 100 & $242.14 \mathrm{t}$ & 265.38 & $\mathrm{p}$ & $300.32 \mathrm{~g}$ & $269.28 \mathbf{E}$ & $19.23 \mathrm{~s}$ & $20.98 \mathrm{n}$ & $22.62 \mathrm{j}$ & $20.94 \mathbf{E}$ \\
\hline $\mathbf{C u}$ & 25 & $200.54 \mathrm{y}$ & 226.14 & $\mathrm{v}$ & $271.52 \mathrm{~h}$ & $232.73 \mathbf{H}$ & $15.10 \mathrm{z}$ & $17.13 \mathrm{w}$ & 20.45 o & $17.56 \mathbf{H}$ \\
\hline $\mathrm{Cu}$ & 50 & $209.56 \mathrm{w}$ & 262.5 & q & $280.16 \mathrm{~d}$ & $250.74 \mathbf{G}$ & $15.28 \mathrm{y}$ & $19.77 \mathrm{r}$ & 21.701 & $18.92 \mathbf{G}$ \\
\hline $\mathbf{F e}+\mathbf{C u}$ & $\mathbf{5 0}+\mathbf{2 5}$ & $247.36 \mathrm{~s}$ & 297.60 & $\mathrm{k}$ & $359.65 \mathrm{e}$ & $301.54 \mathbf{D}$ & $18.68 \mathrm{t}$ & $22.43 \mathrm{k}$ & $27.08 \mathrm{e}$ & $22.73 \mathrm{D}$ \\
\hline $\mathbf{F e}+\mathbf{C u}$ & $\mathbf{5 0}+\mathbf{5 0}$ & $267.70 \quad$ o & 321.54 & $\mathrm{r}$ & $395.36 \mathrm{c}$ & $328.20 \mathrm{C}$ & $20.16 \mathrm{p}$ & $24.21 \mathrm{~g}$ & $29.87 \mathrm{c}$ & $24.75 \mathrm{C}$ \\
\hline $\mathbf{F e}+\mathbf{C u}$ & $100+25$ & $300.54 \mathrm{i}$ & 301.64 & $\mathrm{p}$ & $430.28 \mathrm{~b}$ & 344.15 B & $22.63 \mathrm{i}$ & $22.78 \mathrm{~h}$ & $32.46 \mathrm{~b}$ & 25.96 B \\
\hline $\mathbf{F e}+\mathbf{C u}$ & $100+50$ & $332.14 \mathrm{f}$ & 366.52 & $\mathrm{v}$ & $480.64 \mathrm{a}$ & $393.10 \mathrm{~A}$ & $25.01 \mathrm{f}$ & $27.60 \mathrm{~d}$ & $36.24 \mathrm{a}$ & $29.62 \mathrm{~A}$ \\
\hline Mean A & & $247.54 \mathrm{C}$ & 277.691 & & $338.02 \mathrm{~A}$ & & $18.81 \mathrm{C}$ & $21.16 \mathrm{~B}$ & $25.59 \mathrm{~A}$ & \\
\hline
\end{tabular}

Means followed by the same letters in a column or raw do not differ significantly according to Duncan's New Multiple Range test at $\mathrm{P}=0.05$. 
Middle East J. Agric. Res., 10(2): 548-562, 2021

Table 6: Effect of micro nutrients, bio -stimulators and their interaction on number of leaves/ plant and root length of Marigold (Calendula officinalis L.) during (2019 and 2020) seasons.

\begin{tabular}{|c|c|c|c|c|c|c|c|c|c|}
\hline \multicolumn{2}{|c|}{ Treatments } & \multicolumn{4}{|c|}{ Number of leaves/ plant } & \multicolumn{4}{|c|}{ Root length ( cm) } \\
\hline \multirow{4}{*}{$\begin{array}{l}\text { Micro } \\
\text { nutrients }\end{array}$} & Concentrations & \multicolumn{4}{|c|}{$1^{\text {st }}$ season } & \multicolumn{4}{|c|}{$1^{\text {st }}$ season } \\
\hline & ppm & \multicolumn{3}{|c|}{ Bio -stimulators } & Mean B & \multicolumn{3}{|c|}{ Bio-stimulators } & Mean B \\
\hline & & Control & Yeast & Amino acid & & Control & Yeast & Amino acid & \\
\hline & Control & $153.12 \mathrm{z}$ & $168.45 \mathrm{x}$ & $195.16 \mathrm{t}$ & $172.24 \mathbf{I}$ & $25.18 \mathrm{x}$ & $28.58 \mathrm{u}$ & $30.92 \mathrm{p}$ & $28.23 \mathbf{H}$ \\
\hline $\mathbf{F e}$ & 50 & $180.56 \mathrm{v}$ & $203.14 \mathrm{r}$ & 228.471 & $204.06 \mathbf{G}$ & $29.55 \mathrm{~s}$ & $30.35 \mathrm{r}$ & $32.08 \mathrm{n}$ & $30.66 \mathbf{F}$ \\
\hline $\mathbf{F e}$ & 100 & $195.16 \mathrm{t}$ & $215.31 \mathrm{p}$ & $243.86 \mathrm{j}$ & $218.11 \mathbf{E}$ & $30.92 \mathrm{p}$ & $32.78 \mathrm{k}$ & $34.52 \mathrm{i}$ & $32.74 \mathbf{E}$ \\
\hline $\mathbf{C u}$ & 25 & $153.26 \mathrm{y}$ & $185.32 \mathrm{u}$ & 219.77 о & $186.12 \mathbf{H}$ & $25.21 \mathrm{x}$ & $26.25 \mathrm{w}$ & $29.17 \mathrm{t}$ & 26.88 I \\
\hline $\mathbf{C u}$ & 50 & $172.65 \mathrm{w}$ & $214.53 \mathrm{q}$ & $226.88 \mathrm{~m}$ & $204.69 \mathbf{F}$ & $26.25 \mathrm{w}$ & $28.24 \mathrm{v}$ & 32.681 & $29.06 \mathbf{G}$ \\
\hline $\mathbf{F e}+\mathbf{C u}$ & $50+25$ & $200.31 \mathrm{~s}$ & $243.12 \mathrm{k}$ & $292.95 \mathrm{e}$ & 245.46 D & $31.03 \mathrm{o}$ & $33.34 \mathrm{j}$ & $35.25 \mathrm{~h}$ & $33.21 \mathrm{D}$ \\
\hline $\mathrm{Fe}+\mathrm{Cu}$ & $50+50$ & $220.14 \mathrm{n}$ & $262.98 \mathrm{~h}$ & $322.90 \mathrm{c}$ & $268.67 \mathrm{C}$ & $30.47 \mathrm{q}$ & $36.83 \mathrm{~g}$ & $39.17 \mathrm{e}$ & $35.49 \mathrm{C}$ \\
\hline $\mathbf{F e}+\mathbf{C u}$ & $100+25$ & $246.89 \mathrm{i}$ & $287.74 \mathrm{f}$ & $351.97 \mathrm{~b}$ & 295.54 В & $32.67 \mathrm{~m}$ & $42.15 \mathrm{~d}$ & $42.74 \mathrm{c}$ & 39.19 В \\
\hline $\mathbf{F e}+\mathrm{Cu}$ & $100+50$ & $270.56 \mathrm{~g}$ & $302.12 \mathrm{~d}$ & $393.80 \mathrm{a}$ & $322.16 \mathrm{~A}$ & $36.88 \mathrm{f}$ & $43.17 \mathrm{~b}$ & $44.10 \mathrm{a}$ & $41.38 \mathrm{~A}$ \\
\hline \multirow[t]{3}{*}{ Mean A } & & $199.18 \mathrm{C}$ & $231.41 \mathbf{B}$ & $275.08 \mathrm{~A}$ & & $29.80 \mathrm{C}$ & $33.52 \mathrm{~B}$ & $35.63 \mathrm{~A}$ & \\
\hline & & \multicolumn{4}{|c|}{$2^{\text {nd }}$ season } & \multicolumn{4}{|c|}{$2^{\text {nd }}$ season } \\
\hline & Control & $156.28 \mathrm{z}$ & $186.75 \mathrm{v}$ & $211.80 \mathrm{~s}$ & 184.94 I & $26.90 \mathrm{y}$ & $31.88 \mathrm{t}$ & $34.49 \mathrm{n}$ & $31.09 \mathrm{H}$ \\
\hline $\mathrm{Fe}$ & $\mathbf{5 0}$ & $175.42 \mathrm{x}$ & $216.30 \mathrm{r}$ & 239.701 & $210.47 \mathbf{F}$ & $33.26 \mathrm{~s}$ & $34.21 \mathrm{o}$ & $35.72 \mathrm{~m}$ & $34.40 \mathrm{~F}$ \\
\hline $\mathbf{F e}$ & 100 & $181.65 \mathrm{w}$ & $236.70 \mathrm{~m}$ & $257.10 \mathrm{i}$ & $225.15 \mathbf{E}$ & $34.02 \mathrm{p}$ & $36.84 \mathrm{k}$ & $37.05 \mathrm{j}$ & $35.97 \mathbf{E}$ \\
\hline $\mathbf{C u}$ & 25 & $156.30 \mathrm{z}$ & $206.25 \mathrm{u}$ & $225.00 \mathrm{o}$ & $195.85 \mathbf{H}$ & $26.92 \mathrm{y}$ & $30.45 \mathrm{w}$ & $31.63 \mathrm{~V}$ & 29.67 I \\
\hline $\mathrm{Cu}$ & 50 & $164.21 \mathrm{y}$ & $220.15 \mathrm{q}$ & $229.14 \mathrm{n}$ & $204.50 \mathbf{G}$ & $29.37 \mathrm{x}$ & $31.86 \mathrm{u}$ & $33.90 \mathrm{q}$ & $31.71 \mathrm{G}$ \\
\hline $\mathrm{Fe}+\mathrm{Cu}$ & $50+25$ & $209.55 \mathrm{t}$ & $255.00 \mathrm{k}$ & $308.10 \mathrm{e}$ & $257.55 \mathrm{D}$ & $33.33 \mathrm{r}$ & $39.38 \mathrm{~h}$ & $40.62 \mathrm{f}$ & $37.78 \mathrm{D}$ \\
\hline $\mathbf{F e}+\mathbf{C u}$ & $50+50$ & $221.70 \mathrm{p}$ & $274.65 \mathrm{~h}$ & $321.30 \mathrm{~d}$ & $272.55 \mathrm{C}$ & $34.21 \mathrm{o}$ & $40.51 \mathrm{~g}$ & $42.37 \mathrm{~d}$ & $39.03 \mathrm{C}$ \\
\hline $\mathbf{F e}+\mathbf{C u}$ & $100+25$ & $255.45 \mathrm{j}$ & $291.15 \mathrm{~g}$ & $358.35 \mathrm{~b}$ & 301.65 B & 35.881 & $42.06 \mathrm{e}$ & $43.49 \mathrm{~b}$ & 40.48 B \\
\hline $\mathbf{F e}+\mathbf{C u}$ & $100+50$ & $291.75 \mathrm{f}$ & $321.75 \mathrm{c}$ & $377.40 \mathrm{a}$ & $330.30 \mathbf{A}$ & $38.41 \mathrm{i}$ & $43.16 \mathrm{c}$ & $44.77 \mathrm{a}$ & $42.11 \mathrm{~A}$ \\
\hline Mean A & & $201.37 \mathrm{C}$ & 245.41 B & $280.88 \mathbf{A}$ & & $32.46 \mathrm{C}$ & $36.71 \mathrm{~B}$ & $38.23 \mathrm{~A}$ & \\
\hline
\end{tabular}

Means followed by the same letters in a column or raw do not differ significantly according to Duncan's New Multiple Range test at $\mathrm{P}=0.05$. 
Middle East J. Agric. Res., 10(2): 548-562, 2021

Table 7: Effect of micro nutrients, bio- stimulators and their interaction on fresh and dry weight of roots (g/plant) of Marigold (Calendula officinalis L.) during (2019 and 2020) seasons.

\begin{tabular}{|c|c|c|c|c|c|c|c|c|c|}
\hline \multicolumn{2}{|l|}{ Treatments } & \multicolumn{4}{|c|}{ Fresh weight of roots (g/plant) } & \multicolumn{4}{|c|}{ Dry weight of roots (g/plant) } \\
\hline \multirow{3}{*}{ Micro nutrients } & \multirow{3}{*}{$\begin{array}{c}\text { Concentrations } \\
\text { ppm }\end{array}$} & \multicolumn{4}{|c|}{$1^{\text {st }}$ season } & \multicolumn{4}{|c|}{$1^{\text {st }}$ season } \\
\hline & & \multicolumn{3}{|c|}{ Bio -stimulators (g/l) } & \multirow[t]{2}{*}{ Mean B } & \multicolumn{3}{|c|}{ Bio -stimulators (g/l) } & \multirow[t]{2}{*}{ Mean B } \\
\hline & & Control & Yeast & Amino acid & & Control & Yeast & Amino acid & \\
\hline \multicolumn{2}{|c|}{ Control } & $22.25 \mathrm{x}$ & $23.85 \mathrm{y}$ & $27.61 \mathrm{u}$ & 24.57 I & $3.44 x$ & $4.15 \mathrm{w}$ & $4.69 \mathrm{~s}$ & $4.09 \mathbf{I}$ \\
\hline $\mathbf{F e}$ & 50 & $26.35 \mathrm{v}$ & $28.95 \mathrm{r}$ & $32.34 \mathrm{~m}$ & $29.21 \mathbf{F}$ & $4.46 \mathrm{u}$ & $4.95 \mathrm{r}$ & $5.49 \quad 1$ & $4.97 \mathbf{F}$ \\
\hline $\mathbf{F e}$ & 100 & $28.17 \mathrm{t}$ & $30.80 \mathrm{p}$ & $34.49 \mathrm{~h}$ & $31.15 \mathbf{E}$ & $4.59 \mathrm{t}$ & 5.26 o & $5.84 \mathrm{j}$ & $5.23 \mathbf{E}$ \\
\hline $\mathbf{C u}$ & 25 & $23.19 \mathrm{z}$ & $25.97 \mathrm{w}$ & $31.69 \mathrm{n}$ & $26.95 \mathbf{H}$ & $3.44 \mathrm{x}$ & $4.40 \mathrm{v}$ & $5.38 \mathrm{~m}$ & $4.41 \mathbf{H}$ \\
\hline $\mathbf{C u}$ & 50 & $24.25 \mathrm{x}$ & $30.73 \mathrm{q}$ & 32.411 & $29.13 \mathbf{G}$ & $4.15 \mathrm{w}$ & $5.20 \mathrm{p}$ & $5.53 \mathrm{k}$ & $4.96 \mathbf{G}$ \\
\hline $\mathbf{F e}+\mathbf{C u}$ & $50+25$ & $28.71 \mathrm{~s}$ & $34.40 \mathrm{k}$ & $41.43 \mathrm{e}$ & $34.85 \mathrm{D}$ & $4.98 \mathrm{q}$ & $5.84 \mathrm{j}$ & $7.14 \mathrm{e}$ & $5.99 \mathrm{D}$ \\
\hline $\mathbf{F e}+\mathbf{C u}$ & $\mathbf{5 0}+\mathbf{5 0}$ & 31.18 o & $37.63 \mathrm{~g}$ & $45.70 \mathrm{c}$ & $38.17 \mathrm{C}$ & $5.37 \mathrm{n}$ & $6.69 \mathrm{f}$ & $7.86 \mathrm{c}$ & $6.64 \mathrm{C}$ \\
\hline $\mathrm{Fe}+\mathrm{Cu}$ & $100+25$ & $34.93 \mathrm{i}$ & $34.88 \mathrm{j}$ & $49.87 \mathrm{~b}$ & 39.89 В & $5.98 \mathrm{~h}$ & $5.95 \mathrm{i}$ & $8.57 \mathrm{~b}$ & $6.83 \mathrm{~B}$ \\
\hline $\mathrm{Fe}+\mathrm{Cu}$ & $100+50$ & $38.75 \mathrm{f}$ & $42.47 \mathrm{~d}$ & $55.75 \mathrm{a}$ & $45.66 \mathrm{~A}$ & $6.59 \mathrm{~g}$ & $7.42 \mathrm{~d}$ & $9.97 \mathrm{a}$ & 7.99 A \\
\hline \multirow[t]{2}{*}{ Mean A } & & $28.64 \mathrm{C}$ & 32.19 B & $39.03 \mathrm{~A}$ & & $4.78 \mathrm{C}$ & 5.54 B & $6.72 \mathrm{~A}$ & \\
\hline & & \multicolumn{4}{|c|}{$2^{\text {nd }}$ season } & \multicolumn{4}{|c|}{$2^{\text {nd }}$ season } \\
\hline \multicolumn{2}{|c|}{ Control } & $24.51 \mathrm{y}$ & $24.56 \times$ & $28.46 \mathrm{~s}$ & $25.84 \mathrm{I}$ & $4.20 \mathrm{w}$ & $4.17 \mathrm{x}$ & $4.84 \mathrm{t}$ & $4.40 \mathrm{I}$ \\
\hline $\mathbf{F e}$ & 50 & $26.83 \mathrm{u}$ & $29.46 \mathrm{r}$ & $33.16 \mathrm{k}$ & $29.82 \mathbf{F}$ & $4.56 \mathrm{u}$ & $5.01 \mathrm{r}$ & $5.63 \mathrm{k}$ & $5.07 \mathbf{F}$ \\
\hline $\mathbf{F e}$ & 100 & $28.59 \mathrm{t}$ & $31.34 \mathrm{o}$ & $35.35 \mathrm{j}$ & $31.76 \mathbf{E}$ & $4.86 \mathrm{~s}$ & 5.32 o & $6.01 \mathrm{j}$ & $5.40 \mathbf{E}$ \\
\hline $\mathbf{C u}$ & 25 & $23.66 \mathrm{z}$ & $26.68 \mathrm{v}$ & $31.92 \mathrm{~m}$ & $27.42 \mathbf{H}$ & $4.02 \mathrm{y}$ & $4.53 \mathrm{v}$ & $5.42 \mathrm{~m}$ & $4.66 \mathbf{H}$ \\
\hline $\mathbf{C u}$ & 50 & $24.72 \mathrm{w}$ & $30.97 \mathrm{p}$ & 32.931 & $29.54 \mathbf{G}$ & $4.20 \mathrm{w}$ & $5.26 \mathrm{p}$ & 5.591 & $5.02 \mathbf{G}$ \\
\hline $\mathrm{Fe}+\mathrm{Cu}$ & $50+25$ & $29.88 \mathrm{q}$ & $35.81 \mathrm{~h}$ & $42.27 \mathrm{e}$ & 35.99 D & $5.08 \mathrm{q}$ & $6.08 \mathrm{~h}$ & $7.18 \mathrm{e}$ & $6.11 \mathrm{D}$ \\
\hline $\mathbf{F e}+\mathbf{C u}$ & $\mathbf{5 0}+\mathbf{5 0}$ & $31.58 \mathrm{n}$ & $38.94 \mathrm{~g}$ & $46.47 \mathrm{c}$ & $39.00 \mathrm{C}$ & $5.37 \mathrm{n}$ & $6.62 \mathrm{~g}$ & $7.90 \mathrm{c}$ & $6.63 \mathrm{C}$ \\
\hline $\mathbf{F e}+\mathbf{C u}$ & $100+25$ & $35.46 \mathrm{i}$ & $39.59 \mathrm{f}$ & $51.58 \mathrm{~b}$ & $42.21 \mathbf{B}$ & $6.02 \mathrm{i}$ & $6.73 \mathrm{f}$ & $8.76 \mathrm{~b}$ & $7.17 \mathbf{B}$ \\
\hline $\mathrm{Fe}+\mathrm{Cu}$ & $100+50$ & $39.59 \mathrm{f}$ & $43.64 \mathrm{~d}$ & $59.50 \mathrm{a}$ & $47.58 \mathbf{A}$ & $6.73 \mathrm{f}$ & $7.41 \mathrm{~d}$ & $10.11 \mathrm{a}$ & $8.08 \mathbf{A}$ \\
\hline Mean A & & $29.42 \mathrm{C}$ & 33.44 B & $40.18 \mathrm{~A}$ & & $5.00 \mathrm{C}$ & $5.68 \mathrm{~B}$ & $6.83 \mathbf{A}$ & \\
\hline
\end{tabular}

Means followed by the same letters in a column or raw do not differ significantly according to Duncan's New Multiple Range test at $\mathrm{P}=0.05$. 
Middle East J. Agric. Res., 10(2): 548-562, 2021

Table 8: Effect of micro nutrients, bio -stimulators and their interaction on number of flowers/ plant, flower diameter and vase life of Marigold (Calendula officinalis L.) during (2019 and 2020) seasons.

\begin{tabular}{|c|c|c|c|c|c|c|c|c|c|c|c|c|c|}
\hline \multicolumn{2}{|c|}{ Treatments } & \multicolumn{4}{|c|}{ Number of flowers/ plant } & \multicolumn{4}{|c|}{ Flower diameter (cm) } & \multicolumn{4}{|c|}{ Vase life (Day) } \\
\hline \multirow{3}{*}{$\begin{array}{c}\text { Micro } \\
\text { nutrients }\end{array}$} & \multirow{3}{*}{$\begin{array}{c}\text { Concentrations } \\
\text { ppm }\end{array}$} & \multicolumn{4}{|c|}{$1^{\text {st }}$ season } & \multicolumn{4}{|c|}{$1^{\text {st }}$ season } & \multicolumn{4}{|c|}{$1^{\text {st }}$ season } \\
\hline & & \multicolumn{3}{|c|}{ Bio- stimulators (g/l) } & \multirow[t]{2}{*}{ Mean B } & \multicolumn{3}{|c|}{ Bio- stimulators (g/l) } & \multirow[t]{2}{*}{ Mean B } & \multicolumn{3}{|c|}{ Bio- stimulators (g/l) } & \multirow{2}{*}{$\begin{array}{l}\text { Mean } \\
\text { B }\end{array}$} \\
\hline & & Control & Yeast & $\begin{array}{l}\text { Amino } \\
\text { acid }\end{array}$ & & Control & Yeast & Amino & & Control & Yeast & $\begin{array}{l}\text { Amino } \\
\text { acid }\end{array}$ & \\
\hline & Control & $16.00 \mathrm{r}$ & $26.00 \mathrm{~h}$ & $26.00 \mathrm{~h}$ & $22.67 \mathrm{I}$ & $7.54 \mathrm{~s}$ & $7.59 \mathrm{r}$ & $8.23 \mathrm{o}$ & 7.79 I & $7.03 \mathrm{z}$ & $8.12 \mathrm{t}$ & $8.20 \mathrm{q}$ & $7.78 \mathrm{I}$ \\
\hline $\mathbf{F e}$ & 50 & 24.82 o & 30.46 & $30.46 \mathrm{~g}$ & $28.58 \mathbf{F}$ & 8.24 o & 8.301 & $8.49 \mathrm{fg}$ & $8.34 \mathbf{F}$ & $7.14 \mathrm{y}$ & $8.19 \mathrm{r}$ & 8.32 o & $7.88 \mathbf{H}$ \\
\hline $\mathbf{F e}$ & 100 & $26.46 \mathrm{~m}$ & $32.48 \mathrm{k}$ & $32.48 \mathrm{k}$ & $30.47 \mathbf{E}$ & 8.23 o & $8.28 \mathrm{~m}$ & $8.56 \mathrm{~d}$ & $8.36 \mathbf{E}$ & $7.19 \mathrm{x}$ & $8.26 \mathrm{p}$ & $8.34 \mathrm{n}$ & $7.93 \mathrm{G}$ \\
\hline $\mathbf{C u}$ & 25 & $20.46 \mathrm{q}$ & $29.28 \mathrm{i}$ & $29.28 \mathrm{i}$ & $26.34 \mathbf{H}$ & $8.20 \mathrm{p}$ & $8.18 \mathrm{q}$ & $8.23 \mathrm{o}$ & $8.20 \mathbf{H}$ & $7.30 \mathrm{w}$ & $8.37 \mathrm{~m}$ & 8.461 & $8.04 \mathbf{F}$ \\
\hline $\mathbf{C u}$ & 50 & $22.84 \mathrm{p}$ & $30.24 \mathrm{e}$ & $30.24 \mathrm{e}$ & $27.77 \mathbf{G}$ & $8.35 \mathrm{k}$ & 8.301 & $8.35 \mathrm{k}$ & $8.33 \mathbf{G}$ & $7.40 \mathrm{v}$ & $8.49 \mathrm{k}$ & $8.56 \mathrm{j}$ & $8.15 \mathbf{E}$ \\
\hline $\mathbf{F e}+\mathbf{C u}$ & $50+25$ & 27.041 & $39.00 \mathrm{~d}$ & $39.00 \mathrm{~d}$ & $35.01 \mathrm{D}$ & $8.38 \mathrm{j}$ & $8.36 \mathrm{k}$ & $8.48 \mathrm{~g}$ & $8.41 \mathrm{D}$ & $8.49 \mathrm{k}$ & $8.58 \mathrm{i}$ & $9.14 \mathrm{e}$ & $8.74 \mathrm{D}$ \\
\hline $\mathbf{F e}+\mathbf{C u}$ & $\mathbf{5 0}+\mathbf{5 0}$ & $29.30 \mathrm{j}$ & $43.04 \mathrm{c}$ & $43.04 \mathrm{c}$ & $38.46 \mathrm{C}$ & $8.43 \mathrm{~h}$ & $8.40 \mathrm{i}$ & $8.56 \mathrm{~d}$ & $8.46 \mathrm{C}$ & $8.61 \mathrm{~h}$ & $8.67 \mathrm{~g}$ & $9.24 \mathrm{~d}$ & $8.84 \mathrm{C}$ \\
\hline $\mathbf{F e}+\mathbf{C u}$ & $100+25$ & $32.90 \mathrm{f}$ & $46.92 \mathrm{~b}$ & $46.92 \mathrm{~b}$ & $42.25 \mathrm{~B}$ & $8.58 \mathrm{c}$ & $8.51 \mathrm{e}$ & $8.65 \mathrm{~b}$ & 8.58 B & $8.78 \mathrm{f}$ & $8.03 \mathrm{c}$ & $9.79 \mathrm{~b}$ & $8.87 \mathrm{~B}$ \\
\hline $\mathbf{F e}+\mathbf{C u}$ & $100+50$ & $36.46 \mathrm{n}$ & $52.50 \mathrm{n}$ & $58.50 \mathrm{a}$ & $49.15 \mathrm{~A}$ & $8.26 \mathrm{n}$ & $8.50 \mathrm{ef}$ & $9.03 \mathrm{a}$ & $8.60 \mathrm{~A}$ & $8.14 \mathrm{~s}$ & $9.25 \mathrm{u}$ & $10.04 \mathrm{a}$ & $9.14 \mathrm{~A}$ \\
\hline \multirow[t]{3}{*}{ Mean A } & & $26.25 \mathrm{C}$ & $36.66 \mathrm{~B}$ & $37.32 \mathbf{A}$ & & $8.25 \mathrm{C}$ & $8.27 \mathbf{B}$ & $8.51 \mathbf{A}$ & & $7.79 \mathrm{C}$ & 8.44 B & $8.90 \mathrm{~A}$ & \\
\hline & & \multicolumn{4}{|c|}{$2^{\text {nd }}$ season } & & \multicolumn{2}{|c|}{$2^{\text {nd }}$ season } & \multicolumn{5}{|c|}{$2^{\text {nd }}$ season } \\
\hline & Control & $18.00 \mathrm{z}$ & $24.90 \mathrm{r}$ & $28.24 \mathrm{~s}$ & $23.71 \mathrm{I}$ & $7.15 \mathrm{x}$ & $7.46 \mathrm{w}$ & $8.26 \mathrm{t}$ & $7.62 \mathrm{I}$ & $6.14 \mathrm{y}$ & $7.53 \mathrm{q}$ & $8.23 \mathrm{n}$ & $7.30 \mathbf{I}$ \\
\hline $\begin{array}{l}\mathrm{Fe} \\
\mathrm{Fe}\end{array}$ & $\begin{array}{l}50 \\
100\end{array}$ & $\begin{array}{l}22.04 \mathrm{x} \\
24.22 \mathrm{y}\end{array}$ & $\begin{array}{l}28.84 \mathrm{~m} \\
31.56 \mathrm{n}\end{array}$ & $\begin{array}{l}31.961 \\
34.28 \mathrm{i}\end{array}$ & $\begin{array}{l}27.61 \mathbf{G} \\
30.02 \mathbf{E}\end{array}$ & $\begin{array}{ll}8.26 & \mathrm{t} \\
8.30 \mathrm{~s}\end{array}$ & $\begin{array}{l}8.541 \\
8.56 \mathrm{k}\end{array}$ & $\begin{array}{l}8.56 \mathrm{k} \\
8.63 \mathrm{~h}\end{array}$ & $\begin{array}{ll}8.45 & \mathbf{F} \\
8.50 & \mathbf{E}\end{array}$ & $\begin{array}{l}7.02 \mathrm{x} \\
7.11 \mathrm{w}\end{array}$ & $\begin{array}{l}7.14 \mathrm{v} \\
7.26 \mathrm{u}\end{array}$ & $\begin{array}{l}8.56 \mathrm{i} \\
8.62 \mathrm{~h}\end{array}$ & $\begin{array}{l}7.57 \mathbf{H} \\
7.66 \mathbf{G}\end{array}$ \\
\hline $\mathbf{C u}$ & 25 & $20.84 \mathrm{w}$ & $27.50 \mathrm{v}$ & $30.00 \mathrm{p}$ & $26.11 \mathbf{H}$ & $8.20 \mathrm{v}$ & $8.34 \mathrm{r}$ & $8.4 \mathrm{q}$ & $8.31 \mathbf{H}$ & $7.32 \mathrm{t}$ & $7.34 \mathrm{~s}$ & $8.71 \mathrm{f}$ & $7.79 \mathbf{F}$ \\
\hline $\mathrm{Cu}$ & 50 & $24.62 \mathrm{t}$ & $31.28 \mathrm{o}$ & 31.961 & $29.29 \mathbf{F}$ & $8.23 \mathrm{u}$ & 8.48 o & $8.59 \mathrm{j}$ & $8.43 \mathbf{G}$ & $7.56 \mathrm{p}$ & $7.52 \mathrm{r}$ & $8.81 \mathrm{e}$ & $7.96 \mathbf{E}$ \\
\hline $\mathbf{F e}+\mathbf{C u}$ & $50+25$ & $27.94 \mathrm{q}$ & $34.00 \mathrm{k}$ & $41.08 \mathrm{e}$ & $34.34 \mathrm{D}$ & $8.40 \mathrm{q}$ & $8.61 \mathrm{i}$ & $8.74 \mathrm{e}$ & $8.58 \mathrm{D}$ & 8.12 o & $8.12 \mathrm{o}$ & $9.00 \mathrm{~d}$ & $8.41 \mathrm{D}$ \\
\hline $\mathbf{F e}+\mathbf{C u}$ & $50+50$ & $29.56 \mathrm{j}$ & $36.62 \mathrm{~h}$ & $42.84 \mathrm{~d}$ & $36.34 \mathrm{C}$ & $8.43 \mathrm{p}$ & $8.64 \mathrm{~g}$ & $8.8 \mathrm{c}$ & $8.62 \mathrm{C}$ & $8.23 \mathrm{n}$ & $8.35 \mathrm{~m}$ & $9.15 \mathrm{c}$ & $8.58 \mathrm{C}$ \\
\hline $\mathbf{F e}+\mathbf{C u}$ & $100+25$ & $34.06 \mathrm{f}$ & $38.82 \mathrm{~g}$ & $47.78 \mathrm{~b}$ & 40.22 B & $8.51 \mathrm{n}$ & $8.70 \mathrm{f}$ & $8.84 \mathrm{~b}$ & 8.68 B & $8.45 \mathrm{k}$ & $8.41 \quad 1$ & $9.53 \mathrm{~b}$ & $8.80 \mathrm{~B}$ \\
\hline $\mathbf{F e}+\mathbf{C u}$ & $100+50$ & $38.90 \mathrm{v}$ & $42.90 \mathrm{c}$ & $50.32 \mathrm{a}$ & $44.04 \mathbf{A}$ & $8.53 \mathrm{~m}$ & $8.75 \mathrm{~d}$ & 8.91 a & $8.73 \mathrm{~A}$ & $8.50 \mathrm{j}$ & $8.63 \mathrm{~g}$ & $9.63 \mathrm{a}$ & $8.92 \mathrm{~A}$ \\
\hline Mean A & & $26.69 \mathrm{C}$ & 32.94 B & $37.61 \mathrm{~A}$ & & $8.22 \mathrm{C}$ & 8.45 B & $8.64 \mathrm{~A}$ & & $7.61 \mathrm{C}$ & $7.81 \mathrm{~B}$ & $8.92 \mathbf{A}$ & \\
\hline
\end{tabular}

Means followed by the same letters in a column or raw do not differ significantly according to Duncan's New Multiple Range test at $\mathrm{P}=0.05$. 
Copper at $50 \mathrm{ppm}$ coupled with amino acid at the rate of $2 \mathrm{~g} / 1$ gave the highest increment in all parameters followed by the treatment of Iron at the rate of $50 \mathrm{ppm}$ and Copper at the rate of $25 \mathrm{ppm}$ coupled with amino acid at the rate of $2 \mathrm{~g} / \mathrm{l}$ as compared with control treatment in both seasons, respectively. The rise of all parameters per plant might be due the used fertilizer might play an active role (direct or indirect) in cell division, the application of complete fertilizer which consists of macro and microelements such as nitrogen for its importance to consist the amino acids to form the protein which participate in cell enlargement and cell division. However, phosphorus has an essential role in producing energy for the physiological processes as synthesis of proteins by formation of the coenzyme adenine triphosphate (ATP). Moreover, potassium plays a direct or indirect role in plant metabolism (Devlin, 1979). Many reports showed that micronutrients have important efficiency on growth and enlargement on plants, the promoted effect on all parameters might be due to Fe might play an important role in the prophyrin structure of chlorophyll. In green plants, in other hand, there is often a good correlation between the level of supply and the chlorophyll content. Generally, Iron is one of the three micro essential nutrient elements required by plants. Fe is important in cytochrome structure. Micro elements participate in most of the enzymatic reaction and they also play a role indirectly through the synthesis of several growth regulators, micronutrients such as iron have important roles in plant growth and yield of aromatic and medicinal plants. Iron is also related with the establishment of chlorophyll. $\mathrm{Fe}$ has also a main character in the activation of numerous enzymes. The deficiency of $\mathrm{Fe}$ can cause numerous physiological irregularities such as chlorosis, scorching and resetting etc. Many studies have reported that foliar application of amino acids caused an increase in the growth and development of plants (Schönherr et al. 2005, Naguib et al., 2005, Heidari et al., 2008, Ramrodi, 2011, Omer et al. 2013, Karuppaiah, 2014 and Hashem, 2016).

\subsection{Effect of foliar application of micro nutrients, bio-stimulators and their interaction on chemical constituents in Calendula plants:}

The obtained results of both seasons in Table (9, 10 and 11) revealed that spraying Calendula plants with either Iron at the rate of $100 \mathrm{ppm}$ and Copper at the rate of $50 \mathrm{ppm}$ coupled with amino acid at the rate of $2 \mathrm{~g}$. / 1 gave high significantly increased chemical constituents as compared to the untreated treatment. These results are owing to the use of micronutrients that play an important role in the representation of critical auxins that increase cell division and increase the content chlorophyll in the leaf. Generally, the obtained results revealed that under Ismailia sandy soil conditions applying foliar fertilizer as $\mathrm{Fe}$ at the rate of $100 \mathrm{ppm}$ and $\mathrm{Cu}$ at the rate of $50 \mathrm{ppm}$ with $2 \mathrm{~g} / 1$ amino acid resulted in highest improvement of chemical constituents. These results are in a good harmony with (Yadegari, 2013a and Yadegari, $2016 \mathrm{c}$ ) on marigold declared that foliar application with Fe, Zn or Mn individual or in mixtures affected positively on the above-mentioned records of plant chemical composition. The primitive effect of foliar fertilizers were in agreement with those obtained by (Habib, 2012; Abou-Sreea and Yassen, 2016 and Jan et al., 2017).

\section{Acknowledgment}

The authors would like to thank Prof. Dr. Emam M.S. Nofal Prof. of Floriculture and Ornamental Hort., Horticulture Department, Fac. Agric. Kafr El-Sheikh, Kafr El-Sheikh Univ., Prof. Dr. Dr Bothina weheda, Prof. Dr Samia, Z. El-Bably and Prof. Dr Bothina weheda Prof. Dr. of Floriculture and Ornamental Hort., Horticulture Dept. Horticulture Research Institute for providing facilities, collaboration, continues help and guidance.

\section{Conclusion}

In brief, it could be concluded from the results that iron, copper and amino acid fertilization had a significant effect on Calendula plants .The application of the treatment of $100 \mathrm{ppm}$ of iron and 50 ppm copper coupled with amino acid at the rate of $2 \mathrm{~g} / 1$ improved growth performance, flowering traits and chemical constituents (total chlorophyll in leaves, beta carotenoids, total flavonoids in dry ray flowers, nitrogen, phosphorus and potassium percentage, iron, copper and zinc (ppm) in leaves) as compared with other treatments. 
Table 9: Effect of micro nutrients, bio- stimulators and their interaction on total chlorophyll, beta-carotene and total flavonoids in dry ray flowers and of Marigold (Calendula officinalis L.) during (2019 and 2020) seasons.

\begin{tabular}{|c|c|c|c|c|c|c|c|c|c|c|c|c|c|}
\hline \multicolumn{2}{|c|}{ Treatments } & \multicolumn{4}{|c|}{$\begin{array}{l}\text { Total chlorophyll } \\
\text { (mg g/ g. f.w.) }\end{array}$} & \multicolumn{4}{|c|}{$\begin{array}{l}\text { Beta-carotene in dry ray flowers } \\
\text { (mg g/g d. w.) }\end{array}$} & \multicolumn{4}{|c|}{$\begin{array}{l}\text { Total flavonoids in dry ray flowers } \\
\text { (mg /g D. w.) }\end{array}$} \\
\hline \multirow{3}{*}{$\begin{array}{c}\text { Micro } \\
\text { nutrients }\end{array}$} & \multirow{3}{*}{$\begin{array}{l}\text { Concentrations } \\
(\text { ppm) }\end{array}$} & \multicolumn{4}{|c|}{$1^{\text {st }}$ season } & \multicolumn{4}{|c|}{$1^{\text {st }}$ season } & \multicolumn{4}{|c|}{$1^{\text {st }}$ season } \\
\hline & & \multicolumn{3}{|c|}{ Bio- stimulators (g/l) } & \multirow{2}{*}{ Mean B } & \multicolumn{3}{|c|}{ Bio- stimulators (g/l) } & \multirow[t]{2}{*}{ Mean B } & \multicolumn{3}{|c|}{ Bio- stimulators (g/l) } & \multirow{2}{*}{ Mean B } \\
\hline & & Control & Yeast & $\begin{array}{l}\text { Amino } \\
\text { acid }\end{array}$ & & Control & Yeast & $\begin{array}{l}\text { Amino } \\
\text { acid }\end{array}$ & & Control & Yeast & $\begin{array}{l}\text { Amino } \\
\text { acid }\end{array}$ & \\
\hline & ntrol & $1.04 \mathrm{~W}$ & $1.19 \mathrm{~V}$ & $1.23 \mathrm{t}$ & $1.15 \mathrm{I}$ & $1.36 \mathrm{z}$ & $1.39 \mathrm{y}$ & $1.42 \mathrm{x}$ & $1.39 \mathbf{H}$ & $10.89 \mathrm{z}$ & $13.25 \mathrm{x}$ & $14.64 \mathrm{v}$ & $12.93 \mathrm{I}$ \\
\hline $\mathbf{F e}$ & $\mathbf{5 0}$ & $1.21 \mathrm{u}$ & $1.26 \mathrm{~s}$ & $1.28 \mathrm{r}$ & $1.25 \mathbf{H}$ & $1.63 \mathrm{~s}$ & $1.46 \mathrm{w}$ & $1.54 \mathrm{v}$ & $1.54 \mathbf{G}$ & $13.16 \mathrm{y}$ & $14.68 \mathrm{u}$ & $15.12 \mathrm{t}$ & $14.32 \mathbf{H}$ \\
\hline $\mathbf{F e}$ & 100 & $1.26 \mathrm{~s}$ & $1.31 \mathrm{q}$ & $1.36 \mathrm{o}$ & $1.31 \mathbf{G}$ & $1.64 \mathrm{r}$ & $1.47 \mathrm{v}$ & $1.56 \mathrm{t}$ & $1.56 \mathbf{F}$ & $14.62 \mathrm{w}$ & $15.64 \mathrm{~s}$ & $16.21 \mathrm{q}$ & $15.49 \mathbf{G}$ \\
\hline $\mathbf{C u}$ & 25 & $1.34 \mathrm{p}$ & $1.36 \mathrm{o}$ & $1.41 \mathrm{~m}$ & $1.37 \mathbf{F}$ & $1.76 \mathrm{q}$ & $1.88 \mathrm{j}$ & $1.89 \mathrm{i}$ & $1.84 \mathrm{D}$ & $15.89 \mathrm{r}$ & 18.00 o & $18.65 \mathrm{~m}$ & $17.51 \mathbf{F}$ \\
\hline $\mathbf{C u}$ & 50 & $1.39 \mathrm{n}$ & 1.421 & $1.46 \mathrm{j}$ & $1.42 \mathbf{E}$ & 1.79 о & $1.81 \mathrm{n}$ & $1.86 \mathrm{k}$ & $1.82 \mathbf{E}$ & $16.85 \mathrm{p}$ & $18.45 \mathrm{n}$ & 18.781 & $18.03 \mathbf{E}$ \\
\hline $\mathbf{F e}+\mathbf{C u}$ & $50+25$ & $1.44 \mathrm{k}$ & $1.46 \mathrm{j}$ & $1.52 \mathrm{~h}$ & $1.47 \mathrm{D}$ & 1.79 о & $1.83 \mathrm{~m}$ & 1.851 & $1.82 \mathbf{E}$ & $18.96 \mathrm{k}$ & $19.11 \mathrm{i}$ & $21.03 \mathrm{~g}$ & 19.74 D \\
\hline $\mathbf{F e}+\mathrm{Cu}$ & $\mathbf{5 0}+\mathbf{5 0}$ & $1.51 \mathrm{i}$ & $1.53 \mathrm{~g}$ & $1.59 \mathrm{e}$ & $1.54 \mathrm{C}$ & $1.78 \mathrm{p}$ & $1.97 \mathrm{~h}$ & $2.76 \mathrm{c}$ & $2.17 \mathrm{C}$ & $19.02 \mathrm{j}$ & $21.03 \mathrm{~g}$ & $23.15 \mathrm{c}$ & $21.07 \mathrm{C}$ \\
\hline $\mathrm{Fe}+\mathrm{Cu}$ & $100+25$ & $1.56 \mathrm{f}$ & $1.67 \mathrm{c}$ & $1.69 \mathrm{~b}$ & $1.64 \mathrm{~B}$ & $2.50 \mathrm{~g}$ & $2.57 \mathrm{e}$ & $2.81 \mathrm{~b}$ & $2.63 \mathrm{~B}$ & $19.45 \mathrm{~h}$ & $21.52 \mathrm{e}$ & $24.35 \mathrm{~b}$ & 21.77 B \\
\hline $\mathrm{Fe}+\mathrm{Cu}$ & $100+50$ & $1.62 \mathrm{~d}$ & $1.69 \mathrm{~b}$ & $1.88 \mathrm{a}$ & $1.73 \mathrm{~A}$ & $2.53 \mathrm{f}$ & $2.59 \mathrm{~d}$ & $2.89 \mathrm{a}$ & $2.67 \mathrm{~A}$ & $21.15 \mathrm{f}$ & $22.65 \mathrm{~d}$ & $26.12 \mathrm{a}$ & $22.97 \mathbf{A}$ \\
\hline \multirow[t]{3}{*}{ Mean A } & & $1.37 \mathrm{C}$ & $1.43 \mathrm{~B}$ & $1.49 \mathrm{~A}$ & & $1.86 \mathrm{C}$ & $1.89 \mathrm{~B}$ & $2.06 \mathrm{~A}$ & & $16.55 \mathrm{C}$ & $18.26 \mathbf{B}$ & $19.80 \mathbf{A}$ & \\
\hline & & \multicolumn{4}{|c|}{$2^{\text {nd }}$ season } & & \multicolumn{2}{|c|}{$2^{\text {nd }}$ season } & \multicolumn{5}{|c|}{$2^{\text {nd }}$ season } \\
\hline & ntrol & $1.06 \mathrm{x}$ & $1.28 \mathrm{v}$ & $1.32 \mathrm{t}$ & $1.22 \mathbf{I}$ & $1.23 \mathrm{u}$ & $1.32 \mathrm{u}$ & $1.36 \mathrm{~s}$ & $1.30 \mathbf{H}$ & $10.08 \mathrm{w}$ & $12.36 \mathrm{u}$ & $13.69 \mathrm{r}$ & $12.04 \mathrm{I}$ \\
\hline $\mathrm{Fe}$ & $\mathbf{5 0}$ & $1.22 \mathrm{w}$ & $1.30 \mathrm{u}$ & $1.35 \mathrm{~s}$ & $1.29 \mathbf{H}$ & $1.53 \mathrm{r}$ & $1.62 \mathrm{t}$ & $1.64 \mathrm{~m}$ & $1.60 \mathbf{G}$ & $12.23 \mathrm{v}$ & $13.32 \mathrm{~s}$ & $14.56 \mathrm{p}$ & $13.37 \mathbf{H}$ \\
\hline $\mathbf{F e}$ & 100 & $1.36 \mathrm{r}$ & $1.38 \mathrm{q}$ & $1.42 \mathrm{p}$ & $1.39 \mathbf{G}$ & $1.58 \mathrm{q}$ & $1.64 \mathrm{o}$ & $1.68 \mathrm{j}$ & $1.63 \mathbf{F}$ & $13.21 \mathrm{t}$ & $14.23 \mathrm{q}$ & $15.32 \mathrm{n}$ & $14.25 \mathbf{G}$ \\
\hline $\mathbf{C u}$ & 25 & $1.45 \mathrm{o}$ & $1.48 \mathrm{n}$ & $1.50 \mathrm{~m}$ & $1.48 \quad \mathbf{F}$ & $1.61 \mathrm{p}$ & $1.68 \mathrm{~m}$ & $1.71 \mathrm{i}$ & $1.67 \mathbf{E}$ & $14.56 \mathrm{p}$ & $17.32 \mathrm{k}$ & $16.23 \mathrm{~m}$ & $16.04 \mathbf{F}$ \\
\hline $\mathbf{C u}$ & 50 & 1.511 & $1.56 \mathrm{k}$ & $1.57 \mathrm{j}$ & $1.55 \mathbf{E}$ & $1.63 \mathrm{n}$ & $1.67 \mathrm{j}$ & $1.72 \mathrm{~h}$ & $1.67 \mathbf{E}$ & $15.23 \mathrm{o}$ & $18.65 \mathrm{i}$ & 17.231 & $17.04 \mathbf{E}$ \\
\hline $\mathrm{Fe}+\mathrm{Cu}$ & $50+25$ & $1.62 \mathrm{i}$ & $1.64 \mathrm{~h}$ & $1.68 \mathrm{f}$ & $1.65 \mathrm{D}$ & 1.651 & $1.68 \mathrm{k}$ & $1.76 \mathrm{f}$ & $1.70 \mathrm{D}$ & 17.231 & $19.00 \mathrm{~h}$ & $19.52 \mathrm{~g}$ & $18.58 \mathbf{D}$ \\
\hline $\mathbf{F e}+\mathrm{Cu}$ & $50+50$ & $1.66 \mathrm{~g}$ & $1.69 \mathrm{e}$ & $1.71 \mathrm{~d}$ & $1.69 \mathrm{C}$ & $1.71 \mathrm{i}$ & $1.74 \mathrm{~g}$ & $1.89 \mathrm{c}$ & $1.78 \mathrm{C}$ & $18.23 \mathrm{j}$ & $20.23 \mathrm{f}$ & $20.23 \mathrm{f}$ & $19.56 \mathrm{C}$ \\
\hline $\mathrm{Fe}+\mathrm{Cu}$ & $100+25$ & $1.69 \mathrm{e}$ & $1.71 \mathrm{~d}$ & $1.74 \mathrm{~b}$ & $1.71 \mathrm{~B}$ & $1.89 \mathrm{c}$ & $1.79 \mathrm{e}$ & $1.95 \mathrm{~b}$ & $1.88 \mathrm{~B}$ & $19.52 \mathrm{~g}$ & $22.00 \mathrm{~d}$ & $22.31 \mathrm{c}$ & $21.28 \mathrm{~B}$ \\
\hline $\mathbf{F e}+\mathbf{C u}$ & $100+50$ & $1.72 \mathrm{c}$ & $1.74 \mathrm{~b}$ & $1.78 \mathrm{a}$ & $1.75 \mathrm{~A}$ & $1.89 \mathrm{c}$ & $1.88 \mathrm{~d}$ & $2.13 \mathrm{a}$ & $1.97 \mathbf{A}$ & $20.31 \mathrm{e}$ & $22.36 \mathrm{~b}$ & $25.23 \mathrm{a}$ & $22.63 \mathrm{~A}$ \\
\hline Mean A & & $1.48 \mathrm{C}$ & $1.53 \mathrm{~B}$ & $1.56 \mathrm{~A}$ & & $1.64 \mathrm{C}$ & $1.67 \mathrm{~B}$ & $1.76 \mathrm{~A}$ & & $15.62 \mathrm{C}$ & $17.72 \mathbf{B}$ & $18.26 \mathbf{A}$ & \\
\hline
\end{tabular}

Means followed by the same letters in a column or raw do not differ significantly according to Duncan's New Multiple Range test at $\mathrm{P}=0.05$ 
Middle East J. Agric. Res., 10(2): 548-562, 2021

Table 10: Effect of micro nutrients, bio- stimulators and their interaction on N, P and K of Marigold (Calendula officinalis L.) during (2019 and 2020)

\begin{tabular}{|c|c|c|c|c|c|c|c|c|c|c|c|c|c|}
\hline \multicolumn{2}{|c|}{ Treatments } & \multicolumn{4}{|c|}{$\mathbf{N} \%$} & \multicolumn{4}{|c|}{$\mathbf{P} \%$} & \multicolumn{4}{|c|}{ K\% } \\
\hline \multirow{3}{*}{$\begin{array}{l}\text { Micro } \\
\text { nutrients }\end{array}$} & \multirow{3}{*}{$\begin{array}{c}\text { Concentrations } \\
\text { (ppm) }\end{array}$} & \multicolumn{4}{|c|}{$1^{\text {st }}$ season } & \multicolumn{4}{|c|}{$1^{\text {st }}$ season } & \multicolumn{4}{|c|}{$1^{\text {st }}$ season) } \\
\hline & & \multicolumn{3}{|c|}{ Bio- stimulators (g/l) } & \multirow[t]{2}{*}{ Mean B } & \multicolumn{3}{|c|}{ Bio- stimulators (g/l) } & \multirow[t]{2}{*}{ Mean B } & \multicolumn{3}{|c|}{ Bio -stimulators (g/l) } & \multirow{2}{*}{$\begin{array}{c}\text { Mean } \\
\text { B }\end{array}$} \\
\hline & & Control & Yeast & $\begin{array}{l}\text { Amino } \\
\text { acid }\end{array}$ & & Control & Yeast & $\begin{array}{l}\text { Amino } \\
\text { acid }\end{array}$ & & Control & Yeast & $\begin{array}{l}\text { Amino } \\
\text { acid }\end{array}$ & \\
\hline & Control & $2.20 \mathrm{x}$ & $3.47 \mathrm{w}$ & $3.53 \mathrm{u}$ & $3.07 \quad \mathbf{I}$ & $1.12 \mathrm{~V}$ & $1.18 \mathrm{u}$ & $1.38 \mathrm{p}$ & $1.23 \mathrm{I}$ & $1.92 \mathrm{~V}$ & $2.25 \mathrm{q}$ & $2.40 \mathrm{o}$ & $2.19 \mathrm{I}$ \\
\hline $\mathbf{F e}$ & 50 & $3.61 \mathrm{t}$ & $3.72 \mathrm{p}$ & 4.001 & $3.78 \mathbf{H}$ & $1.20 \mathrm{t}$ & $1.27 \mathrm{~s}$ & $1.47 \mathrm{~m}$ & $1.31 \mathbf{H}$ & $2.10 \mathrm{t}$ & $2.56 \mathrm{n}$ & $2.77 \mathrm{~h}$ & $2.48 \mathbf{G}$ \\
\hline $\mathbf{F e}$ & 100 & $3.68 \mathrm{q}$ & $3.83 \mathrm{n}$ & $4.10 \mathrm{k}$ & $3.87 \mathbf{F}$ & $1.33 \mathrm{r}$ & $1.35 \mathrm{q}$ & 1.531 & $1.40 \mathbf{G}$ & $2.19 \mathrm{r}$ & $2.60 \mathrm{~m}$ & $2.83 \mathrm{f}$ & $2.54 \mathbf{E}$ \\
\hline $\mathrm{Cu}$ & 25 & $3.50 \mathrm{v}$ & $3.63 \mathrm{~s}$ & $4.23 \mathrm{i}$ & $3.79 \mathrm{G}$ & $1.40 \mathrm{o}$ & $1.43 \mathrm{n}$ & $1.62 \mathrm{i}$ & $1.48 \mathbf{F}$ & $2.05 \mathrm{~V}$ & 2.621 & $2.70 \mathrm{j}$ & $2.46 \mathrm{H}$ \\
\hline $\mathbf{C u}$ & 50 & $3.60 \mathrm{t}$ & $3.69 \mathrm{q}$ & $5.31 \mathrm{~g}$ & $4.20 \mathbf{E}$ & $1.48 \mathrm{~m}$ & $1.56 \mathrm{k}$ & $1.73 \mathrm{f}$ & $1.59 \mathbf{E}$ & $2.12 \mathrm{~s}$ & $2.70 \mathrm{j}$ & $2.75 \mathrm{i}$ & $2.52 \mathrm{~F}$ \\
\hline $\mathbf{F e}+\mathbf{C u}$ & $50+25$ & $3.65 \mathrm{r}$ & $4.15 \mathrm{j}$ & $5.81 \mathrm{~d}$ & $4.54 \mathrm{D}$ & 1.521 & $1.59 \mathrm{j}$ & $1.84 \mathrm{~d}$ & $1.65 \mathrm{D}$ & $2.24 \mathrm{q}$ & $2.80 \mathrm{~g}$ & $2.99 \mathrm{~d}$ & $2.68 \mathrm{D}$ \\
\hline $\mathrm{Fe}+\mathrm{Cu}$ & $\mathbf{5 0}+\mathbf{5 0}$ & $3.72 \mathrm{p}$ & $4.52 \mathrm{~h}$ & $6.30 \mathrm{c}$ & $4.85 \mathrm{C}$ & $1.56 \mathrm{k}$ & $1.63 \mathrm{hi}$ & $1.91 \mathrm{c}$ & $1.70 \mathrm{C}$ & $2.27 \mathrm{p}$ & $2.89 \mathrm{e}$ & $3.06 \mathrm{c}$ & $2.74 \mathrm{C}$ \\
\hline $\mathbf{F e}+\mathbf{C u}$ & $100+25$ & $3.75 \mathrm{o}$ & $5.61 \mathrm{f}$ & $6.40 \mathrm{~b}$ & $5.25 \mathrm{~B}$ & $1.64 \mathrm{~h}$ & $1.68 \mathrm{~g}$ & $1.97 \mathrm{~b}$ & $1.76 \mathrm{~B}$ & $2.59 \mathrm{~m}$ & $3.00 \mathrm{~d}$ & $3.10 \mathrm{~b}$ & $2.90 \mathrm{~B}$ \\
\hline $\mathbf{F e}+\mathbf{C u}$ & $100+50$ & $3.85 \mathrm{~m}$ & $5.72 \mathrm{e}$ & $6.50 \mathrm{a}$ & $5.36 \mathrm{~A}$ & $1.69 \mathrm{~g}$ & $1.76 \mathrm{e}$ & $2.00 \mathrm{a}$ & $1.82 \mathbf{A}$ & $2.67 \mathrm{k}$ & $3.07 \mathrm{c}$ & $3.20 \mathrm{a}$ & $2.98 \mathrm{~A}$ \\
\hline \multirow[t]{3}{*}{ Mean A } & & $3.51 \mathrm{C}$ & $4.26 \mathbf{B}$ & $5.13 \mathrm{~A}$ & & $1.44 \mathrm{C}$ & 1.49 B & $1.72 \mathrm{~A}$ & & $2.24 \mathrm{C}$ & 2.72 B & $2.87 \mathrm{~A}$ & \\
\hline & & \multicolumn{4}{|c|}{$2^{\text {nd }}$ season } & & \multicolumn{2}{|c|}{$2^{\text {nd }}$ season } & \multicolumn{5}{|c|}{$2^{\text {nd }}$ season } \\
\hline & Control & $3.02 \mathrm{x}$ & $3.30 \mathrm{w}$ & $3.45 \mathrm{v}$ & $3.26 \quad$ I & $0.96 \mathrm{~s}$ & $1.12 \mathrm{r}$ & $1.25 \mathrm{n}$ & $1.11 \mathrm{I}$ & $1.75 \mathrm{w}$ & $2.10 \mathrm{v}$ & $2.35 \mathrm{p}$ & $2.07 \quad \mathrm{I}$ \\
\hline $\mathbf{F e}$ & 50 & $3.52 \mathrm{u}$ & $3.60 \mathrm{~s}$ & 3.861 & $3.66 \mathrm{H}$ & $1.12 \mathrm{r}$ & $1.30 \mathrm{~m}$ & $1.35 \mathrm{k}$ & $1.26 \mathbf{H}$ & $2.14 \mathrm{u}$ & $2.58 \mathrm{o}$ & 2.691 & $2.47 \mathrm{H}$ \\
\hline $\mathbf{F e}$ & 100 & $3.62 \mathrm{r}$ & 3.75 no & $4.12 \mathrm{j}$ & $3.83 \mathbf{F}$ & $1.16 \mathrm{p}$ & 1.321 & $1.42 \mathrm{j}$ & $1.30 \mathbf{F}$ & $2.22 \mathrm{~s}$ & $2.62 \mathrm{n}$ & $2.72 \mathrm{k}$ & $2.52 \mathbf{F}$ \\
\hline $\mathrm{Cu}$ & 25 & $3.54 \mathrm{t}$ & $3.61 \mathrm{rs}$ & $4.26 \mathrm{i}$ & $3.80 \mathbf{G}$ & $1.14 \mathrm{q}$ & $1.25 \mathrm{n}$ & $1.46 \mathrm{i}$ & $1.28 \mathbf{G}$ & $2.15 \mathrm{u}$ & $2.65 \mathrm{~m}$ & $2.74 \mathrm{j}$ & $2.51 \mathrm{G}$ \\
\hline $\mathbf{C u}$ & 50 & $3.62 \mathrm{r}$ & $3.64 \mathrm{q}$ & $4.36 \mathrm{~g}$ & $3.87 \mathbf{E}$ & $1.20 \mathrm{o}$ & $1.29 \mathrm{~m}$ & $1.62 \mathrm{f}$ & $1.37 \mathbf{E}$ & $2.18 \mathrm{t}$ & $2.76 \mathrm{i}$ & $2.78 \mathrm{~h}$ & $2.57 \mathbf{E}$ \\
\hline $\mathrm{Fe}+\mathrm{Cu}$ & $50+25$ & $3.67 \mathrm{p}$ & $4.00 \mathrm{k}$ & $5.02 \mathrm{f}$ & $4.23 \mathrm{D}$ & $1.43 \mathrm{j}$ & $1.47 \mathrm{i}$ & $1.65 \mathrm{e}$ & $1.52 \mathrm{D}$ & $2.26 \mathrm{r}$ & $2.84 \mathrm{~g}$ & $2.86 \mathrm{f}$ & $2.65 \mathrm{D}$ \\
\hline $\mathrm{Fe}+\mathrm{Cu}$ & $\mathbf{5 0}+50$ & 3.74 o & $4.30 \mathrm{~h}$ & $5.20 \mathrm{~d}$ & $4.41 \mathrm{C}$ & $1.52 \mathrm{~g}$ & $1.49 \mathrm{~h}$ & $1.72 \mathrm{c}$ & $1.58 \mathrm{C}$ & $2.28 \mathrm{q}$ & $2.86 \mathrm{f}$ & $3.02 \mathrm{e}$ & $2.72 \mathrm{C}$ \\
\hline $\mathbf{F e}+\mathbf{C u}$ & $100+25$ & $3.76 \mathrm{n}$ & $5.41 \quad b$ & $5.26 \mathrm{c}$ & $4.81 \mathrm{~B}$ & $1.60 \mathrm{f}$ & $1.62 \mathrm{f}$ & $1.74 \mathrm{~b}$ & $1.65 \mathrm{~B}$ & $2.64 \mathrm{~m}$ & $3.08 \mathrm{~d}$ & $3.20 \mathrm{~b}$ & 2.97 B \\
\hline $\begin{array}{l}\mathrm{Fe}+\mathrm{Cu} \\
\text { Mean A }\end{array}$ & $100+50$ & $\begin{array}{l}3.80 \mathrm{~m} \\
3.59 \mathrm{C}\end{array}$ & $\begin{array}{l}5.53 \mathrm{a} \\
4.13 \mathrm{~B}\end{array}$ & $\begin{array}{l}5.14 \mathrm{e} \\
4.52 \mathrm{~A}\end{array}$ & $4.82 \mathbf{A}$ & $\begin{array}{l}1.65 \mathrm{e} \\
1.31 \mathrm{C}\end{array}$ & $\begin{array}{l}1.69 \mathrm{~d} \\
1.39 \mathrm{~B}\end{array}$ & $\begin{array}{l}1.81 \mathrm{a} \\
1.56 \mathrm{~A}\end{array}$ & $1.72 \mathrm{~A}$ & $\begin{array}{l}2.691 \\
2.26 \mathrm{C}\end{array}$ & $\begin{array}{l}3.10 \mathrm{c} \\
2.73 \mathrm{~B}\end{array}$ & $\begin{array}{l}3.22 \mathrm{a} \\
2.84 \mathbf{A}\end{array}$ & $3.00 \mathrm{~A}$ \\
\hline
\end{tabular}

Means followed by the same letters in a column or raw do not differ significantly according to Duncan's New Multiple Range test at $\mathrm{P}=0.05$. 
Middle East J. Agric. Res., 10(2): 548-562, 2021

Table 11: Effect of micro nutrients, bio - stimulators and their interaction on Fe, Cu and Mn of Marigold (Calendula officinalis L.) during (2019 and 2020)

\begin{tabular}{|c|c|c|c|c|c|c|c|c|c|c|c|c|c|}
\hline \multicolumn{2}{|c|}{ Treatments } & \multicolumn{4}{|c|}{ Fe (ppm) } & \multicolumn{4}{|c|}{ Cu (ppm) } & \multicolumn{4}{|c|}{ Mn (ppm) } \\
\hline \multirow{3}{*}{$\begin{array}{l}\text { Micro } \\
\text { nutrients }\end{array}$} & \multirow{3}{*}{$\begin{array}{c}\text { Concentrations } \\
\text { (ppm) }\end{array}$} & \multicolumn{4}{|c|}{$1^{\text {st }}$ season } & \multicolumn{4}{|c|}{$1^{\text {st }}$ season } & \multicolumn{4}{|c|}{$1^{\text {st }}$ season) } \\
\hline & & \multicolumn{3}{|c|}{ Bio- stimulators (g/l) } & \multirow[t]{2}{*}{ Mean B } & \multicolumn{3}{|c|}{ Bio -stimulators (g/l) } & \multirow[t]{2}{*}{ Mean B } & \multicolumn{3}{|c|}{ Bio-stimulators (g/l) } & \multirow{2}{*}{$\begin{array}{c}\text { Mean } \\
\text { B }\end{array}$} \\
\hline & & Control & Yeast & $\begin{array}{l}\text { Amino } \\
\text { acid }\end{array}$ & & Control & Yeast & $\begin{array}{l}\text { Amino } \\
\text { acid }\end{array}$ & & Control & Yeast & $\begin{array}{l}\text { Amino } \\
\text { acid }\end{array}$ & \\
\hline & Control & $154.23 \mathrm{z}$ & $165.42 \mathrm{q}$ & $172.43 \mathrm{~m}$ & $164.03 \mathrm{I}$ & $22.15 \mathrm{z}$ & $23.15 \mathrm{~g}$ & $24.23 \mathrm{r}$ & $23.18 \mathrm{I}$ & $22.06 \mathrm{y}$ & $23.14 \mathrm{x}$ & $24.06 \mathrm{u}$ & $23.09 \mathrm{I}$ \\
\hline $\mathbf{F e}$ & 50 & $172.26 \mathrm{v}$ & $185.23 \mathrm{n}$ & $190.20 \mathrm{c}$ & $182.56 \mathbf{F}$ & $24.03 x$ & $24.65 \mathrm{u}$ & $26.11 \mathrm{w}$ & $24.93 \mathbf{~ H}$ & $23.14 \mathrm{x}$ & $23.65 \mathrm{v}$ & $25.60 \mathrm{~m}$ & $24.13 \mathbf{~ H}$ \\
\hline $\mathbf{F e}$ & 100 & $178.25 \mathrm{~s}$ & $189.65 \mathrm{t}$ & $193.15 \mathrm{u}$ & $187.02 \mathbf{E}$ & $24.52 \mathrm{v}$ & $24.96 \mathrm{q}$ & $27.15 \mathrm{k}$ & $25.54 \mathbf{G}$ & $23.19 \mathrm{w}$ & $26.52 \mathrm{j}$ & $29.15 \mathrm{i}$ & $26.29 \mathbf{G}$ \\
\hline $\mathbf{C u}$ & 25 & $162.52 \mathrm{y}$ & $175.24 \mathrm{n}$ & $187.23 \mathrm{~m}$ & $175.00 \mathbf{H}$ & $25.15 \mathrm{t}$ & $25.65 \mathrm{~s}$ & $28.64 \mathrm{~h}$ & $26.48 \mathbf{F}$ & $22.05 \mathrm{z}$ & $24.61 \mathrm{p}$ & $33.02 \mathrm{f}$ & $26.56 \mathrm{~F}$ \\
\hline $\mathbf{C u}$ & 50 & $168.52 \mathrm{w}$ & $179.74 \mathrm{t}$ & 191.231 & $179.83 \mathbf{G}$ & $25.89 q$ & $26.12 \mathrm{p}$ & $28.17 \mathrm{i}$ & $26.73 \mathbf{E}$ & $24.14 \mathrm{t}$ & $24.18 \mathrm{~s}$ & $33.12 \mathrm{e}$ & $27.15 \mathbf{E}$ \\
\hline $\mathbf{F e}+\mathbf{C u}$ & $50+25$ & 189.00 о & $191.65 \mathrm{r}$ & $200.36 \mathrm{e}$ & $193.67 \mathbf{D}$ & $26.13 \mathrm{o}$ & 26.961 & $29.15 \mathrm{f}$ & $27.41 \mathrm{D}$ & $24.33 \mathrm{r}$ & 26.021 & $35.12 \mathrm{~d}$ & $28.49 \mathrm{D}$ \\
\hline $\mathrm{Fe}+\mathrm{Cu}$ & $\mathbf{5 0}+\mathbf{5 0}$ & $192.31 \mathrm{j}$ & $195.35 \mathrm{k}$ & $205.15 \mathrm{~d}$ & $197.60 \mathrm{C}$ & $26.78 \mathrm{n}$ & $26.89 \mathrm{~m}$ & $30.23 \mathrm{c}$ & $27.97 \mathrm{C}$ & $24.45 \mathrm{q}$ & $26.14 \mathrm{k}$ & $38.17 \mathrm{c}$ & $29.59 \mathrm{C}$ \\
\hline $\mathrm{Fe}+\mathrm{Cu}$ & $100+25$ & $195.41 \mathrm{~h}$ & $200.25 \mathrm{~g}$ & $210.30 \mathrm{~b}$ & 201.42 B & $27.23 \mathrm{j}$ & $27.64 \mathrm{j}$ & $34.00 \mathrm{~b}$ & 29.62 B & 24.650 & $32.14 \mathrm{~g}$ & $41.15 \mathrm{~b}$ & $32.65 \mathrm{~B}$ \\
\hline $\mathrm{Fe}+\mathrm{Cu}$ & $100+50$ & $200.25 \mathrm{f}$ & $205.56 \mathrm{f}$ & $215.00 \mathrm{a}$ & 206.94 A & $29.12 \mathrm{~g}$ & $30.12 \mathrm{e}$ & $35.00 \mathrm{a}$ & $31.41 \mathrm{~A}$ & $25.19 \mathrm{n}$ & $32.11 \mathrm{~h}$ & $48.12 \mathrm{a}$ & $35.14 \mathrm{~A}$ \\
\hline \multirow[t]{3}{*}{ Mean A } & & $179.19 \mathrm{C}$ & $187.38 \mathbf{B}$ & $196.12 \mathbf{A}$ & & $25.67 \mathrm{C}$ & 26.24 B & $29.19 \mathbf{A}$ & & $25.19 \mathrm{C}$ & $32.11 \mathrm{~B}$ & $48.12 \mathrm{~A}$ & \\
\hline & & \multicolumn{4}{|c|}{$2^{\text {nd }}$ season } & & \multicolumn{2}{|c|}{$2^{\text {nd }}$ season } & \multicolumn{5}{|c|}{$2^{\text {nd }}$ season } \\
\hline & Control & $139.42 \mathrm{z}$ & $145.23 \mathrm{y}$ & $159.32 \mathrm{w}$ & $147.99 \mathbf{~ H}$ & $21.30 \mathrm{z}$ & $23.45 \mathrm{y}$ & $24.36 \mathrm{w}$ & $23.04 \quad$ I & $21.20 \mathrm{y}$ & $24.00 \mathrm{u}$ & 24.86 o & $23.35 \mathbf{I}$ \\
\hline $\mathrm{Fe}$ & 50 & $164.23 \mathrm{u}$ & $168.12 \mathrm{t}$ & $173.23 \mathrm{o}$ & $168.53 \mathbf{E}$ & $24.15 \mathrm{x}$ & $24.52 \mathrm{~V}$ & $24.65 \mathrm{u}$ & $24.44 \mathbf{H}$ & $22.23 x$ & $24.53 \mathrm{~s}$ & $25.65 \mathrm{k}$ & $24.18 \mathbf{H}$ \\
\hline $\mathbf{F e}$ & 100 & $168.32 \mathrm{r}$ & $174.21 \mathrm{n}$ & 178.501 & $173.68 \mathbf{G}$ & $24.65 \mathrm{u}$ & $24.71 \mathrm{t}$ & $24.83 \mathrm{~s}$ & $24.73 \mathbf{G}$ & $22.35 \mathrm{w}$ & $24.62 \mathrm{r}$ & $26.28 \mathrm{i}$ & $24.33 \mathbf{G}$ \\
\hline $\mathrm{Cu}$ & 25 & $153.23 \mathrm{x}$ & $164.23 \mathrm{u}$ & $170.20 \mathrm{q}$ & $162.55 \mathbf{F}$ & $25.36 \mathrm{r}$ & $25.56 \mathrm{q}$ & $25.84 \mathrm{p}$ & $25.59 \mathbf{F}$ & $23.12 \mathrm{~V}$ & $24.96 \mathrm{n}$ & $32.26 \mathrm{f}$ & $26.78 \mathrm{~F}$ \\
\hline $\mathbf{C u}$ & 50 & $162.32 \mathrm{v}$ & $168.23 \mathrm{~s}$ & $172.23 \mathrm{p}$ & $167.59 \mathrm{D}$ & $25.97 \mathrm{o}$ & $26.03 \mathrm{n}$ & $26.15 \mathrm{~m}$ & $26.05 \mathbf{E}$ & $24.53 \mathrm{~s}$ & $24.42 \mathrm{t}$ & $33.12 \mathrm{e}$ & $27.36 \mathbf{E}$ \\
\hline $\mathbf{F e}+\mathbf{C u}$ & $50+25$ & $175.26 \mathrm{~m}$ & $185.25 \mathrm{i}$ & $186.23 \mathrm{~g}$ & $182.25 \mathrm{C}$ & 26.541 & $26.59 \mathrm{k}$ & $26.69 \mathrm{j}$ & $26.61 \mathrm{D}$ & $24.62 \mathrm{r}$ & $25.32 \mathrm{~m}$ & $34.12 \mathrm{~d}$ & $28.02 \mathrm{D}$ \\
\hline $\mathbf{F e}+\mathbf{C u}$ & $50+50$ & $179.25 \mathrm{k}$ & $186.41 \mathrm{f}$ & $188.14 \mathrm{e}$ & 184.60 B & $27.42 \mathrm{i}$ & $27.91 \mathrm{~h}$ & $27.94 \mathrm{~g}$ & $27.76 \mathrm{C}$ & $24.69 \mathrm{q}$ & $26.25 \mathrm{j}$ & $36.12 \mathrm{c}$ & $29.02 \mathrm{C}$ \\
\hline $\mathbf{F e}+\mathbf{C u}$ & $100+25$ & $184.25 \mathrm{j}$ & $190.00 \mathrm{~d}$ & $195.20 \mathrm{~b}$ & $189.82 \mathbf{A}$ & $28.54 \mathrm{f}$ & $28.65 \mathrm{e}$ & $28.89 \mathrm{~d}$ & 28.69 B & $24.70 \mathrm{p}$ & $29.35 \mathrm{~h}$ & $40.26 \mathrm{~b}$ & 31.44 B \\
\hline $\mathrm{Fe}+\mathrm{Cu}$ & $100+50$ & $\begin{array}{l}185.32 \mathrm{~h} \\
16796 \mathrm{C}\end{array}$ & $\begin{array}{l}194.52 \mathrm{c} \\
175\end{array}$ & $196.23 \mathrm{a}$ & $192.02 \mathrm{~A}$ & $29.35 \mathrm{c}$ & $30.54 \mathrm{~b}$ & $33.42 \mathrm{a}$ & $31.10 \mathrm{~A}$ & 25.631 & $30.12 \mathrm{~g}$ & $45.13 \mathrm{a}$ & $33.63 \mathrm{~A}$ \\
\hline Mean A & & $167.96 \mathrm{C}$ & 175.13 B & $179.92 \mathbf{A}$ & & $25.92 \mathrm{C}$ & 26.44 B & $26.97 \mathbf{A}$ & & $23.67 \mathrm{C}$ & 25.94 B & $33.09 \mathrm{~A}$ & \\
\hline
\end{tabular}

Means followed by the same letters in a column or raw do not differ significantly according to Duncan's New Multiple Range test at $\mathrm{P}=0.05$. 


\section{References}

Abou-Sreea, A.I.B. and A.A. Yassen, 2016. Supplying Calendula Plants with some micronutrients as foliar spray under Egyptian soils features. International Journal of Pharm. Tech Research, 9(12): 097-108.

Ashwlayan V.D., A. Kumar, M. Verma, V.K. Garg and S.K. Gupta, 2018. Therapeutic potential of Calendula officinalis. Pharm Pharmacol Int. J., 6(2):149-155.

Association of Official Agricultural Chemists AOAC, 1970. Official methods analysis. pp. 159- 211 (10th End) AOAC, Washington, C.D.

Bacot, A.M., 1954. Chemical composition of representative grands of the 1952 and 1954 crops of the crude tobacco. U.S. Government printing office" Washington 1960.

Bashir, S., K.H. Janbaz and Q. Jabeen, 2006. Studies on spasm genic and spasmolytic activities of Calendula Officinal are flowers. Phytother Res., 20:906-910.

Bolderston, A., N.S.L. Loyd, and R.K. Wong, 2006. The prevention and management of acute skin reactions related to radiation therapy: a systematic review and practice guideline. Support Care Cancer, 14:802-817.

Borghei, M., R. Arjmandiand and R. Moogouei, 2011. Potential of Calendula alata for phytoremediation of stable cesium and lead from solutions. Environmental Monitoring Assessment, 181: 63-68.

Broadley, M.R., P.J. White, J.P. HammondI and Z.A. Lux, 2007. Zinc in plants. New Phytologist, 173:677-702.

Bunghez, I.R. and R.M. Ion, 2011. Complex spectral characterization of active principles from marigold (Calendula officinalis). Journal of Science and Arts, 1:59-64.

Cottenie A.M., L. Verloo, G.V. Kiekens and R. Camerlynck, 1982. Chemical Analysis of Plant and Soil, $100-129$.

Devlin, R.M., 1979. Plant Physiology. Third eddition. Afiliated East-West. Press Prt Lrd. Newdelhy Madrass.

Fonseca, Y.M., C.D. Catini, A. Vicentini, N.R.F. Gerlach and M.J.V. Fonseca, 2010. Protective effect of $C$. officinalis extract against UVB-induced oxidative stress in skin: Evaluation of reduced glutathione levels and matrix metalloproteinase secretion. J. Ethnopharmacol., 127: 596-601.

Habib, M., 2012. Effect of supplementary nutrition with Fe, Zn chelates and urea on wheat quality and quantity. African Journal of Biotechnology, 11: 2661-2665.

Hamburger, M., S. Adler, and D. Baumann, 2003. Preparative purification of the major antiinflammatory triterpenoid esters from Marigold (Calendula officinalis). Fitoterapia, 74(4):328338.

Hashem, Hanan, A.E., 2016. Effect of Sowing Date and Fertilization Treatments on Growth and Chemical Constituents of Calendula officinalis Plants under North Sinai conditions. Middle East J. Agric. Res., 5(4): 761-774.

Hassegawa, R.H., H. Fonseca, A.L. Fancelli, V.N. da Silva, E.A. Schammass, T.A. Reis, and B. Corre`a, 2008. Influence of macro-and micro nutrient fertilization on fungal contamination and fumonisin production in corn grains. Food Control, 19: 36-43.

Heidari, F., S. Zehtab, A. Javanshir and M. Dadpour, 2008. The effect of micronutrient and density on yield and essence production of Menthapiperita L. Iranian journal of medicinal and aromatic plants, 24(1): 1-9.

Hussain, A., G. Nabil, M. Ilyas, M.N. Khan, W. Khan, S. Zeb, M. Hilal, Y. Ali and A. Khan, 2020. Effect of zinc and iron on growth, flowering and shelf life of marigold under the agro-climatic conditions of Sawabi. Pure Appl. Biol., 9(1): 180-192.

Jan, N., K.I. Andrabi and R. John, 2017. Calendula officinalis - an important medicinal plant with potential biological properties. Proc Indian Natn. Sci. Acad., 83 (4):769-787.

Karuppaiah, P., 2014. Effect of zinc and iron on growth, yield and quality of chrysanthemum (Dendrathemum grandiflorum Tzeuleu) The Asian Journal of Horticulture. 9: 1: 232-236.

Khan, M.U., B.R. Ankur, A. Deepika, R. Shadan, S. Seema and H. Ansari, 2011. Diverse belongings of Calendula officinalis: An overview. International Journal of Pharmaceutical Sciences and Drug Research; 3(3): 173-177. 
Klute, A., 1986. Part 1. Physical and mineralogical methods.ASA-SSSA- Agronomy, Madison, Wisconsin USA.

Moran, R., 1982. Formula for determination of chlorophyllous pigments with N, N-dimethylformamide. Plant Physiol., 69: 1376-1381.

MSTATC Computer Program, 1985. Software Program for Design,

Naguib, N.Y., M.Y. Khalil and S.E. El Sherbeny, 2005. A Comparative study on the productivity and chemical constituents of various sources and species of Calendula Plants as affected by two foliar fertilizers. Journal of Applied Sciences Research, 1(2): 176-189.

Nofal, Fayza H., M.U. El-Segai and A. Engy Seleem, 2015. Response of Calendula officinalis L. Plants to Growth Stimulants under Salinity Stress. American-Eurasian J. Agric. and Environ. Sci., 15 (9): 1767-1778.

Omer, E.A., H.A.H. Said-Al Ahl, A.G. El Gendy, Kh. A. Shaban and M.S. Hussein, 2013. Effect of amino acids application on production, volatile oil and chemical composition of chamomile cultivated in saline soil at Sinai. J. Appl. Sci. Res., 9(4): 3006-3021.

Page, A.L., R.H. Millar and D.R. Keeney, 1982. Methods of soil analysis part I and II (Agron., 9) Soil Sci. Soc. Amer., Inc., Madison Wisconsim, U.S.A.

Rafiee, H., A. Mehrafarin, A. Qaderi, S. KalateJari, and H. NaghdiBadi, 2013. Phytochemical, agronomical and morphological responses of Pot Marigold (Calendula officinalis L.) to foliar application of bio-stimulators (Bioactive Amino Acid Compounds). J Nov. Appl Sci., 4 (10): 1100-1103.

Rai, V.K., 2002. Role of amino acids in plant responses to stresses. Biologia Plantarum, 45(4): 481487.

Ramrodi, M., 2011. Evaluation of foliar application of micronutrient and irrigation periods on quantity and quality properties of Plantago ovata Forsk. Iranian Journal of Agriculture of Ecology, 3(2): 223-230.

Schönherr, J., V. Fernandez., and L. Schreiber, 2005. Rates of circular penetration of chelated Fe III: role of humidity, concentration, adjuvants, temperature, and type of chelate. Journal of Agriculture and Food Chemistry, 53: 4484- 4492.

Shahram, Y. F., P. Moradi, and M.Y. Rad, 2015. Effect of foliar application of methanol and chelated zinc on the quantities and qualities yield of Marigold (Calendula officinalis L.). J. Appl. Environ. Biol. Sci., 4(12S) 170-176.

Singh, J.P., K. Kumar and P.N. Katiyar, 2012. Effect of zinc, iron and copper on yield parameters of gladiolus. Hort. Flora Res. Spect., 1(1): 64-68.

Snedecor, G.W. and W.G. Cochran, 1980. Statistical Methods, 7th ed. Iowa State Univ. Press, Ames, Iowa, U.S.A.

Soltanpour, P.N., 1985. Use of ammonium bicarbonate DTPA soil test to evaluate elemental availability and toxicity. Commun Soil Sci Plant Anal 16, 323-338. DOI:10.1080/00103628509367607

Sunitha, H.M., 2006. Effect of plant population, nutrition, pinching and growth regulators on plant growth, seed yield and quality of African marigold. M. Sc. (Hort) Thesis, Univ Agric Sci, Dharward, India.

Ukiya, M., T. Akihisa, K. Yasukawa, H. Tokuda, T. Suzuki and Y. Kimura, 2006. Anti-inflammatory, anti-tumor-promoting, and cytotoxic activities of constituents of marigold (C. officinalis) flowers. J. Nat. Prod., 69: 1692-1696.

Yadegari, M., 2013 a. Foliar Application of $\mathrm{Fe}, \mathrm{Cu}, \mathrm{Mn}$ and B on growth, yield, and essential oil yield of marigold (Calendula officinalis). Journal of Applied Science and Agriculture, 8(5): 559-567.

Yadegari, M., 2013 b. Effect of foliar application of $\mathrm{Fe}, \mathrm{Zn}, \mathrm{Cu}$ and $\mathrm{Mn}$ on yield and essential oils of Borago (Boragoo fficinalis L.). Journal of Applied Science and Agriculture, 8(5): 568-575.

Yadegari, M., 2016 c. Phyto-Chemical and Morphological Characters of Calendula officinalis as affected by micronutrients. Journal of Ornamental Plants, 6(4): 271-278.

Yoshikawa, M.T., A. Kishi, T. Kageura and M. Matsuda, 2001. Medicinal flowers. III Marigold (1): hypoglycemic, gastric emptying inhibitory, and gastro-protective principles and new oleanane type triterpene oligoglycosides, Calendula officinalis. Chem Pharm. Bull. (Tokyo) 49:863-870.

Younis, A., A. Riaz, M. Sajid, N. Mushtaq, M. Ahsan, and M. Nadeem, 2013. Foliar application of macro- and micronutrients on the yield and quality of Rosa hybrid cvs. Cardinal and Whisky Mac. African Journal of Biotechnology, 12(7): 702-708. 